\title{
HÖLDER DOMAINS AND POINCARÉ DOMAINS
}

\author{
WAYNE SMITH AND DAVID A. STEGENGA
}

Abstract. A domain $D \subset R^{d}$ of finite volume is said to be a $p$-Poincaré domain if there is a constant $M_{p}(D)$ so that

$$
\int_{D}\left|u-u_{D}\right|^{p} d x \leq M_{p}^{p}(D) \int_{D}|\nabla u|^{p} d x
$$

for all functions $u \in C^{1}(D)$. Here $u_{D}$ denotes the mean value of $u$ over $D$. Techniques involving the quasi-hyperbolic metric on $D$ are used to establish that various geometric conditions on $D$ are sufficient for $D$ to be a $p$-Poincaré domain. Domains considered include starshaped domains, generalizations of John domains and Hölder domains. $D$ is a Hölder domain provided that the quasi-hyperbolic distance from a fixed point $x_{0} \in D$ to $x$ is bounded by a constant multiple of the logarithm $m_{1}$ of the euclidean distance of $x$ to the boundary of $D$. The terminology is derived from the fact that in the plane, a simply connected Hölder domain has a Hölder continuous Riemann mapping function from the unit disk onto $D$. We prove that if $D$ is a Hölder domain and $p \geq d$, then $D$ is a $p$-Poincaré domain. This answers a question of Axler and Shields regarding the image of the unit disk under a Hölder continuous conformal mapping. We also consider geometric conditions which imply that the imbedding of the Sobolev space $W^{1, p}(D) \rightarrow L^{p}(D)$ is compact, and prove that this is the case for a Hölder domain $D$.

\section{INTRODUCTION}

We consider proper open connected subdomains $D$ of euclidean $d$-space $R^{d}, d \geq 2$. Following [GO] we define the quasi-hyperbolic metric $k_{D}$ in $D$ by

$$
k_{D}\left(x_{1}, x_{2}\right)=\inf _{\gamma} \int_{\gamma} \frac{d s}{\delta_{D}(x)}
$$

where the infimum is taken over all rectifiable arcs $\gamma$ joining $x_{1}$ to $x_{2}$ in $D$. Here we denote by $\delta_{D}(x)$ the euclidean distance between $x$ and $\partial D$.

Fix a point $x_{0} \in D$. We say that $D$ is a Hölder domain if

$$
k_{D}\left(x_{0}, x\right) \leq c_{1} \log \frac{\delta_{D}\left(x_{0}\right)}{\delta_{D}(x)}+c_{2}, \quad x \in D,
$$

Received by the editors October 18, 1988.

1980 Mathematics Subject Classification (1985 Revision). Primary 46E35 Secondary 30C55, 30C60, 35Q99, 42B99.

Key words and phrases. Conformal mapping, quasi-hyperbolic geometry, Poincaré inequality, Sobolev spaces, Whitney decomposition.

First author is supported in part by a grant from the National Science Foundation. 
holds for some finite constants $c_{1}, c_{2}$. Our terminology is motivated by a result of Becker and Pommerenke.

Suppose that $D$ is a bounded simply connected domain in $R^{2}$. A theorem in [BP] implies that $D$ is a Hölder domain if and only if there is a Riemann mapping function $g$ satisfying the Hölder condition

$$
\left|g\left(z_{1}\right)-g\left(z_{2}\right)\right| \leq c\left|z_{1}-z_{2}\right|^{\alpha}, \quad\left|z_{1}\right| \leq 1, \quad\left|z_{2}\right| \leq 1
$$

for some $\alpha>0$. In fact, if $k_{D}$ is replaced by the comparable hyperbolic metric then the exponent $\alpha=c_{1}^{-1}$. See [SS1] for a localization of this. In [GM] Gehring and Martio established an $R^{d}$ version of the Becker and Pommerenke result by showing that a domain $D$ is the image of a ball under a Hölder continuous $k$-quasiconformal mapping if and only if condition (1.2) holds. Domains satisfying (1.2) are said to satisfy a quasi-hyperbolic boundary condition in [GM].

On the otherhand, Hölder domains are closely related to the BMO-Sobolev extension domains studied by Jones in [J1], [J2] and uniform domains. In [GO], Gehring and Osgood show that Jones' extension domains are equivalent to uniform domains. Another closely related type of domain is the John domain. Fix a point $x_{0} \in D$, we say that $D$ is a John domain provided that for each $x_{1} \in D$ there is an arc $\gamma$ joining $x_{0}$ to $x_{1}$ in $D$ along which

$$
\delta_{D}(x) \geq \alpha\left|\gamma\left(x, x_{1}\right)\right|, \quad x \in \gamma .
$$

Here $\alpha$ is a positive constant, $\gamma\left(x, x_{1}\right)$ is the portion of $\gamma$ joining $x$ to $x_{1}$ and $\left|\gamma\left(x, x_{1}\right)\right|$ is its arc length.

The definition of uniform domains given in [GO] shows that uniform domains are John domains, but not conversely. An elementary exercise shows that John domains are Hölder domains. But the thickness condition (1.4), which can be visualized as a twisted cone condition, does not hold in general for Hölder domains. In [SS1], an example of a Hölder domain is constructed which contains a sequence of tubes of width $\varepsilon_{n}>0$ and length $\varepsilon_{n} \log \varepsilon_{n}^{-1}$ where $\varepsilon_{n}$ tends to zero. Thus, (1.4) is violated and hence Hölder domains are not necessarily John domains. See also the example in [BP].

Our interest in Hölder domains is motivated by a question of Axler and Shields [AS]. Suppose that $g$ is a Riemann mapping function mapping the unit disk onto $D \subset R^{2}$ and satisfying (1.3). They asked whether $D$ necessarily satisfied the analytic Poincaré inequality

$$
\iint_{D}|F|^{2} d x d y \leq M \iint_{D}\left|F^{\prime}\right|^{2} d x d y
$$

whenever $F$ is holomorphic in $D$ and vanishes at $g(0) \in D$. Here $M$ is a finite constant. Our main result provides an affirmative answer to this question. This result was known to be true provided the $\alpha$ in (1.3) was greater than $\frac{1}{2}-\frac{1}{320}$.

Let $D \subset R^{d}$ be a domain with finite volume, $m(D)<\infty$, and $1 \leq p<\infty$. We denote by $W^{1, p}(D)$ the usual Sobolev space of functions on $D$ that together 
with their first order weak partial derivatives are in $L^{p}(D)$. The norm for $W^{1, p}(D)$ is given by

$$
\|u\|_{W^{1, p}(D)}=\left(\int_{D}|u|^{p} d x+\int_{D}|\nabla u|^{p} d x\right)^{1 / p} .
$$

We say that $D$ is a $p$-Poincaré domain provided

$$
\sup _{u} \frac{\int_{D}\left|u-u_{D}\right|^{p} d x}{\int_{D}|\nabla u|^{p} d x}=M_{p}^{p}(D)<\infty
$$

holds, where the supremum is taken over all nonconstant functions $u \in W^{1, p}(D)$. Here $u_{D}$ denotes the average of $u$ over $D, u_{D}=\frac{1}{m(D)} \int u d x$. Meyers and Serrin [MS] have shown that $C^{1}(D)$ is dense in $W^{1, p}(D)$, so one only needs to consider functions in $C^{1}(D)$ to establish that a domain $D$ is a $p$-Poincaré domain. Hamilton $[\mathrm{H}]$ has shown that for simply connected planar domains $D$ of finite area, the analytic Poincare inequality (1.5) is equivalent to (1.6) for $p=2$.

Therefore, the Axler-Shields question is answered by our main results:

Theorem 1. If $D \subset R^{d}$ is a Hölder domain, then $D$ is a p-Poincaré domain for all $p \geq d$.

Theorem 2. If a domain $D$ is a Hölder domain, then

$$
\int_{D} k_{D}^{p}\left(x_{0}, x\right) d x<\infty
$$

for all $p<\infty$.

The restriction $p \geq d$ is necessary, as we show by an example at the end of $\S 10$. Nevertheless, it is surprising compared to a recent result of Martio [Mar] where he proves that John domains are $p$-Poincaré domains for all $p \geq 1$. On the otherhand, this restriction compares favorably with a result of Staples [S] that $L^{p}$-averaging domains are $p$-Poincare domains for $p \geq d$. In fact, condition (1.7) implies that Hölder domain are $L^{p}$-averaging domains for all $p \geq 1$, see [S]. The proofs of Theorem 1 and Theorem 2 appear in $\S 4$, while preliminary work is contained in $\S \S 2$ and 3.

$\S \S 5,6$ and 7 contain additional conditions which are shown to be sufficient for the Poincare inequality to hold for a domain $D$. We show in $\S 5$ that a bounded starshaped domain is a $p$-Poincaré domain for $p \geq i$, while in $\S 7$ generalizations of John domains that have cusps are considered. In $\S 6$ a Whitney decomposition of the domain $D$ along with a family of curves in $D$ is used to obtain an estimate of $M_{p}(D)$. This estimate involves integration over the "shadow" of an arbitrary Whitney cube with respect to the curve family. In $\S 7$ we introduce the $k_{p}$ metric, which is a generalization of the quasi-hyperbolic metric, and we use this in our study of $p$-Poincare domains.

The imbedding $W^{1, p}(D) \rightarrow L^{p}(D)$ is studied in $\S 8$. We show that some of our sufficient conditions for the $p$-Poincaré inequality to hold actually imply 
the stronger result that this imbedding is compact. In particular this is shown to be the case when $D$ is a Hölder domain. An example showing that this is not true in general is given in $\S 9$.

The final section concerns a class of domains with simple geometry. This class contains the "rooms and corridors" type domains that have been used by several authors to study the Poincaré inequality. In $\S 10$ we use the $k_{p}$ metric to provide a complete description of such domains for which the Poincare inequality holds, and we partially characterize those for which the imbedding $W^{1, p}(D) \rightarrow L^{p}(D)$ is compact.

\section{LENGTHS OF GEODESICS IN HöLDER DOMAINS}

Let $D$ be a Hölder domain. Definition (1.2) does not make it clear that $D$ is even a bounded domain. Let $x_{1} \in D$, then by Lemma 1 in [GO] there exists a quasi-hyperbolic geodesic $\gamma$ joining $x_{0}$ to $x_{1}$. Thus, $\gamma$ is a rectifiable arc in $D$ and

$$
k_{D}\left(y_{1}, y_{2}\right)=\int_{\gamma\left(y_{1}, y_{2}\right)} \frac{d s}{\delta_{D}(x)}
$$

for each pair of points $y_{1}, y_{2} \in \gamma$. We will show that these geodesics are bounded in length by a multiple of $\delta_{D}\left(x_{0}\right)$.

Theorem 3. Suppose that $D$ satisfies (1.2). Then there is a finite constant $c_{3}$ so that whenever $\gamma$ is a quasi-hyperbolic geodesic joining $x_{0}$ to $x_{1}$ in $D$, the inequality

$$
k_{D}\left(x_{0}, x\right) \leq c_{1} \log \frac{\delta_{D}\left(x_{0}\right)}{\left|\gamma\left(x, x_{1}\right)\right|}+c_{3}
$$

holds for all $x \in \gamma$.

Proof. Fix $x_{1} \in D$ and let $\gamma$ be a quasi-hyperbolic geodesic joining $x_{0}$ to $x_{1}$ in $D$. Assume that $(2.1)$ is false, so that for every $c_{3}<\infty$, there exists an $a_{0} \in \gamma$ with $L=\left|\gamma\left(a_{0}, x_{1}\right)\right|$ and satisfying

$$
c_{1} \log \frac{\delta_{D}\left(x_{0}\right)}{L}+c_{3}<k_{D}\left(x_{0}, a_{0}\right) .
$$

We will show that this is impossible if $c_{3}$ is sufficiently large.

Define $a_{k} \in \gamma\left(a_{k-1}, x_{1}\right)$ by $\left|\gamma\left(a_{k-1}, a_{k}\right)\right|=2^{-k} L$, where $k=1,2, \ldots$. Let $\lambda_{k}=\sup \left\{\delta_{D}(x) / L \mid x \in \gamma\left(a_{k}, x_{1}\right)\right\}$, where $k \geq 0$. Combining (1.2) and (2.2) we get that

$$
c_{1} \log \frac{\delta_{D}\left(x_{0}\right)}{L}+c_{3}<c_{1} \log \frac{\delta_{D}\left(x_{0}\right)}{\delta_{D}(x)}+c_{2}
$$

for all $x \in \gamma\left(a_{0}, x_{1}\right)$ and hence that $\lambda_{0} \leq \exp \left(\frac{c_{2}-c_{3}}{c_{1}}\right)$. We choose $c_{3}$ large enough so that $c_{3}>c_{2}$ and $\lambda_{0}<1 / 2$. 
We prove by induction that $\lambda_{k-1} \leq \lambda_{0}^{k}$. This is trivially true for $k=1$ so assume that it is true for some $k \geq 1$. If $x \in \gamma\left(a_{k}, x_{1}\right)$, then by (2.2)

$$
\begin{aligned}
c_{1} \log \frac{\delta_{D}\left(x_{0}\right)}{L}+c_{3}+\frac{2^{-k}}{\lambda_{k-1}} & \leq \int_{\gamma\left(x_{0}, a_{0}\right)} \frac{d s}{\delta_{D}(y)}+\int_{\gamma\left(a_{k-1}, a_{k}\right)} \frac{d s}{\delta_{D}(y)} \\
& \leq \int_{\gamma\left(x_{0}, x\right)} \frac{d s}{\delta_{D}(y)}=k_{D}\left(x_{0}, x\right) \\
& \leq c_{1} \log \frac{\delta_{D}\left(x_{0}\right)}{\delta_{D}(x)}+c_{2}
\end{aligned}
$$

and hence

$$
\frac{c_{1}^{-1}}{\left(2 \lambda_{0}\right)^{k}} \leq \frac{c_{1}^{-1} 2^{-k}}{\lambda_{k-1}} \leq \log \frac{1}{\lambda_{k}}
$$

Now, $c_{3}$ can be chosen (depending only on $c_{1}$ ) so that $\lambda_{0}$ is so small that

$$
\log \frac{1}{\lambda_{0}^{k+1}} \leq \frac{c_{1}^{-1}}{\left(2 \lambda_{0}\right)^{k}}, \quad k \geq 1 .
$$

Combining (2.3) and (2.4) we see that $\lambda_{k} \leq \lambda_{0}^{k+1}$ and hence the induction hypothesis is satisfied. Since $0<\delta_{D}\left(x_{1}\right) \leq L \lambda_{k} \leq L \lambda_{0}^{k+1}$ holds for all $k \geq 1$ and $\lambda_{0}<1$ we have a contradiction which proves the theorem.

Corollary 1. Suppose that $D$ is Hölder domain and that $\gamma$ is a quasi-hyperbolic geodesic joining $x_{0}$ to $x_{1}$ in $D$. Then

(a) There is a constant $c_{4}=c_{4}\left(c_{1}, c_{2}\right)$ so that $|\gamma| \leq c_{4} \delta_{D}\left(x_{0}\right)$. In particular, $D$ is bounded.

(b) There is a constant $c_{5}=c_{5}\left(c_{1}, c_{2}\right)$ so that whenever $\gamma_{1}$ is a subarc of $\gamma$ and $L=\left|\gamma_{1}\right| / 2 \delta_{D}\left(x_{0}\right)$, then

$$
\frac{L}{\log \frac{1}{L}} \leq c_{5} \max \left\{\frac{\delta_{D}(x)}{\delta_{D}\left(x_{0}\right)} \mid x \in \gamma_{1}\right\} .
$$

Proof. The proof of (a) follows immediately from (2.1). To prove (b), assume that $\gamma_{1}=\gamma(a, c)$ and that $b \in \gamma_{1}$ satisfies $|\gamma(a, b)|=\left|\gamma_{1}\right| / 2$. Let $\lambda=\max \left\{\delta_{D}(x) / \delta_{D}\left(x_{0}\right) \mid x \in \gamma_{1}\right\}$. It follows from (2.1) that

$$
\frac{L}{\lambda}=\frac{\left|\gamma_{1}\right|}{2 \lambda \delta_{D}\left(x_{0}\right)} \leq k(a, b) \leq c_{1} \log \frac{2 \delta_{D}\left(x_{0}\right)}{\left|\gamma_{1}\right|}+c_{3}
$$

and hence that

$$
\frac{L}{\log \frac{1}{L}} \leq\left[c_{1}+\frac{c_{3}}{\log \frac{1}{L}}\right] \lambda \leq\left[c_{1}+\frac{c_{3}}{\log \frac{2}{c_{4}}}\right] \lambda=c_{5} \lambda
$$

which is $(2.5)$. This completes the proof. 
We observe that (2.5) places a restriction on the length of a tube in $D$. If a tube has a diameter of $\varepsilon$, then its length is $O\left(\varepsilon \log \varepsilon^{-1}\right)$.

\section{THE EXPONENTIAL INTEGRABILITY OF $k_{D}$ IN JOHN DOMAINS}

Let $W=\{Q\}$ be a Whitney decomposition of $D$ into closed dyadic cubes with disjoint interiors. This means that the coordinates of the vertices of each cube are dyadic rational numbers and that the diameter of each cube $Q \in W$, which we denote by $d(Q)$, is comparable to its distance to $\partial D$. See Chapter 6 of Stein's book [St] for the existence of such a decomposition. Let $x_{0}$ be the center of some fixed cube $Q_{0} \in W$. Suppose that $Q \in W, Q \neq Q_{0}$ and that $x_{Q}$ is the center of $Q$. If $x \in Q$, then $k_{D}\left(x_{0}, x\right) / k_{D}\left(x_{0}, x_{Q}\right)$ is bounded from above and from below by positive constants (depending on $W$, which in turn only depends on $d$ ). We use the notation $a \approx b$ and $a \preceq b$ to denote that $a$, $b$ are either comparable or satisfy an inequality with a constant depending only on the dimension $d$.

Lemma 1. Suppose that $a_{n}>0$ and that

$$
\sum_{k=n}^{\infty} a_{k} \leq c a_{n}, \quad n=1,2, \ldots,
$$

then $a_{n} \leq 2 c a_{1} \exp (-n / 2 c)$.

Proof. We compute for $k \geq 1$,

$$
\begin{aligned}
\sum_{n=1}^{\infty} n^{k} a_{n} & \leq k \sum_{n=1}^{\infty} a_{n} \sum_{m=1}^{n} m^{k-1}=k \sum_{m=1}^{\infty} m^{k-1} \sum_{n=m}^{\infty} a_{n} \\
& \leq c k \sum_{m=1}^{\infty} m^{k-1} a_{m}
\end{aligned}
$$

and hence $\sum n^{k} a_{n} \leq c^{k+1} k ! a_{1}$. Therefore,

$$
\sum_{n=1}^{\infty} e^{n / 2 c} a_{n} \leq \sum_{k=0}^{\infty} \frac{c a_{1}}{2^{k}} \leq 2 c a_{1}
$$

and the result follows.

In order to provide a clearer picture of our proof of Theorem 2 we first consider the simpler case of John domains. The following theorem can also be derived using results in [MV].

Theorem 4. If $D$ is a John domain, then

$$
\int_{D} e^{\tau k_{D}\left(x_{0}, x\right)} d x<\infty
$$

for some $\tau>0$.

Proof. Assume that (1.4) holds and put $\beta=\delta_{D}\left(x_{0}\right) / \alpha$. Then $|\gamma| \leq \beta$ for all arcs $\gamma$ satisfying (1.4). Since $W$ is a Whitney decomposition we can choose 
$a>0$ so that

$$
a \cdot \max \left(d\left(Q_{1}\right), d\left(Q_{2}\right)\right)<\min \left(d\left(Q_{1}\right), d\left(Q_{2}\right)\right)
$$

whenever $Q_{1}, Q_{2} \in W$ and $Q_{1} \cap Q_{2}$ is nonempty. Let $D_{n}=\bigcup\left\{Q \in W \mid a^{n}<\right.$ $\left.d(Q) / 2 \beta \leq a^{n-1}\right\}$ for $n=1,2, \ldots$.

Since $D \subset B\left(x_{0}, \beta\right)$ we see that $D$ is the disjoint union of the $D_{n}$ 's and by making the $a$ in (3.3) smaller we may assume that $x_{0} \in D_{1}$. Suppose that $x_{1} \in \bigcup_{n}^{\infty} D_{k}$ and that $\gamma$ is an arc in $D$ joining $x_{0}$ to $x_{1}$ along which (1.4) holds. Because of (3.3), there must be a point $x \in \gamma$ and a cube $Q \in W$ with $x \in Q \subset D_{n}$. Since $\delta_{D}(x) \approx \delta_{D}\left(x_{Q}\right)$, there is a dilation $\tilde{Q}$ of $Q$ with $m(\tilde{Q}) \approx m(Q) / \alpha^{d}$ and so that $x_{1} \in \tilde{Q}$.

Let $\tilde{D}_{n}=\bigcup\left\{\tilde{Q} \mid Q \in W, Q \subset D_{n}\right\}$. Then we have shown that

$$
\sum_{k=n}^{\infty} m\left(D_{k}\right)=m\left(\bigcup_{n}^{\infty} D_{k}\right) \leq m\left(\tilde{D}_{n}\right) \preceq \frac{m\left(D_{n}\right)}{\alpha^{d}} .
$$

Applying Lemma 1 we see that

$$
m\left(D_{n}\right) \leq c \varepsilon^{n}, \quad n=1,2, \ldots,
$$

for some constant $c$ and $0<\varepsilon<1$. Since $D$ must also be a Hölder domain we have that for $x \in Q \subset D_{n}$,

$$
k_{D}\left(x_{0}, x\right) \leq c_{1} \log \frac{\delta_{D}\left(x_{0}\right)}{\delta_{D}(x)}+c_{2} \leq c_{1} \log \frac{2 \beta}{d(Q)}+c_{3} \leq c_{4} n
$$

where the $c_{i}$ 's are appropriate constants.

Finally, combining the above estimates we obtain that

$$
\int_{D} e^{\tau k_{D}\left(x_{0}, x\right)} d x=\sum_{n=0}^{\infty} \int_{D_{n}} e^{\tau k_{D}\left(x_{0}, x\right)} d x \leq \sum_{n=0}^{\infty} c e^{\tau c_{4} n} \varepsilon^{n}<\infty
$$

provided $\tau$ is sufficiently small. This completes the proof.

In [J1] it is essentially shown that $m(\partial D)=0$ whenever $D$ is a John domain. Later Martio and Vourinen showed the stronger result that the Hausdorff dimension of $\partial D$ is less than $d$. Carl Sundberg observed that this result follows from Theorem 4 and we thank him for allowing us to include this corollary.

Corollary 2 [MV, Corollary 6.4]. If $D$ is a John domain, then the Hausdorff dimension of $\partial D$ is less than $d$.

Proof. Assume that $D$ is a John domain with fixed point $x_{0} \in D$ and $\alpha$ satisfying (1.4). We cover $\partial D$ with a collection of balls $B\left(y_{1}, r_{1}\right), \ldots, B\left(y_{n}, r_{n}\right)$, where each $y_{i} \in \partial D$. Assume that $r_{i}$ is small enough so that $x_{0} \notin B\left(y_{i}, 3 r_{i}\right)$. Now it follows from (1.4) that there is a ball $B\left(x_{i}, \alpha r_{i}\right) \subset B\left(y_{i}, 3 r_{i}\right) \cap D$. Hence $m\left(B\left(y_{i}, 3 r_{i}\right) \cap D\right) \approx r_{i}^{d}$.

From Lemma 2.1 in [GP] we have

$$
\left|\log \frac{\delta_{D}\left(x_{0}\right)}{\delta_{D}(x)}\right| \leq k_{D}\left(x_{0}, x\right), \quad x \in D,
$$


and it follows that

$$
r_{i}^{-\tau} \leq\left(\frac{3}{\delta_{D}\left(x_{0}\right)}\right)^{\tau} e^{\tau k_{D}\left(x_{0}, x\right)}, \quad x \in B\left(y_{i}, 3 r_{i}\right) \cap D .
$$

By a standard covering lemma, we can find disjoint balls $\left\{B\left(y_{n_{i}}, 3 r_{n_{i}}\right)\right\}$ whose triples cover $\partial D$. Integrating (3.5) over $B\left(y_{n_{i}}, 3 r_{n_{i}}\right) \cap D$ yields that

$$
\begin{aligned}
\sum_{i}\left(9 r_{n_{i}}\right)^{d-\tau} & \preceq \sum_{i} \int_{B\left(y_{n_{i}}, 3 r_{n_{i}}\right) \cap D} e^{\tau k_{D}\left(x_{0}, x\right)} d x \\
& \leq \int_{D} e^{\tau k_{D}\left(x_{0}, x\right)} d x .
\end{aligned}
$$

By the theorem, $\tau>0$ can be chosen small enough so that this last integral is convergent. Hence the dimension of $\partial D$ is no greater than $d-\tau$ and the proof is complete.

\section{The $L^{p}$-INTEgRability of $K_{D}$ IN Hölder domains}

We continue to assume that $W$ is a Whitney decomposition of $D$ with $x_{0}$ the center of some cube $Q_{0}$. In order to prove Theorem 2 and consequently Theorem 1, we modify the argument given in $\S 3$ for John domains. The idea is that while $D$ is not a John domain, it still will have $\delta_{D}(x)$ comparable to $\left|\gamma\left(x, x_{1}\right)\right|$ at many points along a quasi-hyperbolic geodesic $\gamma$ joining $x_{0}$ to $x_{1}$ in $D$.

We start with a modification of Lemma 1 where the right-hand side is replaced with an average.

Lemma 2. Suppose that $a_{k} \geq 0$ and that

$$
\sum_{k=4 n}^{\infty} a_{k} \leq \frac{c_{1}}{n} \sum_{k=n}^{4 n-1} a_{k}, \quad n=1,2, \ldots
$$

for a finite constant $c_{1}$. Then there is a finite constant $c_{2} \preceq c_{1}$ so that for all $p \geq 1$ :

$$
\sum_{k=1}^{\infty} k^{p} a_{k} \leq c_{2}^{p} 2^{p^{2}}\left(a_{1}+a_{2}+a_{3}\right) .
$$

Proof. Let $b_{n}=\sum\left\{a_{k} \mid 4^{n} \leq k<4^{n+1}\right\}$ for $n=0,1, \ldots$. By hypothesis,

$$
b_{n} \leq \sum_{k=4^{n}}^{\infty} a_{k} \leq \frac{c_{1}}{4^{n-1}} b_{n-1}, \quad n>0
$$

For integers $p>0$, we therefore have that

$$
\sum_{k=0}^{\infty} 4^{p k} b_{k} \leq b_{0}+c_{1} \sum_{k=1}^{\infty} 4^{p k+1-k} b_{k-1} \leq 4^{p+1} c_{1} \sum_{k=0}^{\infty} 4^{(p-1) k} b_{k}
$$


assuming that $c_{1} \geq 1$. Hence

$$
\sum_{k=0}^{\infty} k^{p} a_{k} \leq \sum_{n=0}^{\infty} 4^{(n+1) p} b_{n} \leq 4^{p} c_{1}^{p+1} 2^{(p+1)(p+2)} b_{0} \leq 2^{p^{2}} c_{2}^{p} b_{0}
$$

and the proof is complete.

Lemma 3. Suppose that $D$ is a Hölder domain, that $x_{1} \in D$ and that $\gamma$ is a quasi-hyperbolic geodesic joining $x_{0}$ to $x_{1}$ in $D$. If $\delta_{D}\left(x_{1}\right) \leq \delta_{D}\left(x_{0}\right) 2^{-3 n}$ for some integer $n \geq 1$, then there are $n$ distinct integers $m_{1}, \ldots, m_{n}$, with $n \leq m_{i} \leq 3 n$, and points $y_{1}, \ldots, y_{n}$ in $D$ so that

$$
\left|\gamma\left(y_{i}, x_{1}\right)\right| \approx 2^{-m_{i}} \delta_{D}\left(x_{0}\right), \quad\left|\delta_{D}\left(y_{i}\right)\right| \approx 2^{-m_{i}} \delta_{D}\left(x_{0}\right)
$$

Proof. Assume that $\delta_{D}\left(x_{0}\right)=1$. Choose consecutive points $a_{0}, \ldots, a_{2 n}$ satisfying

$$
\left|\gamma\left(a_{m}, x_{1}\right)\right|=2^{-(n+m)}, \quad m=0, \ldots, 2 n .
$$

This is always possible because if $|\gamma|<2^{-n}$, then $1=\delta_{D}\left(x_{0}\right) \leq 2^{-n}+2^{-3 n}<1$. Let $I_{m}$ be the interval $\gamma\left(a_{m-1}, a_{m}\right)$ for $m=1, \ldots, 2 n$.

Suppose that $\delta_{D}(x) \leq \beta\left|\gamma\left(x, x_{1}\right)\right|$ for all $x \in I_{m}$ where $m=m_{1}, \ldots, m_{n}$ and the $\left\{m_{i}\right\}$ are distinct integers in the interval $[1,2 n]$. Using Theorem 3 , it follows that

$$
\begin{aligned}
\frac{n}{\beta} \log 2 & =\sum_{i=1}^{n} \int_{2^{-n-m_{i}}}^{2^{-n-m_{i}+1}} \frac{d s}{\beta s} \leq \sum_{i=1}^{n} \int_{I_{m_{i}}} \frac{d s}{\delta_{D}(x)} \\
& <k_{D}\left(x_{0}, a_{2 n}\right) \leq c_{1} \log \left|\gamma\left(a_{2 n}, x_{1}\right)\right|^{-1}+c_{3} \leq c_{4} n
\end{aligned}
$$

and hence that $\beta>c_{4}^{-1} \log 2$. In otherwords, if we choose $\beta=c_{4}^{-1} \log 2$, then we cannot find $n$ of these intervals.

Thus, there must be $n$ distinct intervals $I_{m_{1}}, \ldots, I_{m_{n}}$ which contain a point $y_{i}$ satisfying $\delta_{D}\left(y_{i}\right) \geq \beta\left|\gamma\left(y_{i}, x_{1}\right)\right|$. We therefore have a lower bound for $\delta_{D}\left(y_{i}\right)$ which is comparable to $2^{-n-m_{i}}$. On the other hand, we have that $\delta_{D}\left(y_{i}\right)$ can not exceed $\left|\gamma\left(y_{i}, x_{1}\right)\right|+\delta_{D}\left(x_{1}\right) \preceq 2^{-n-m_{i}}$. This proves (4.3) and the proof is complete.

Proof of Theorem 2. We continue to assume that $\delta_{D}\left(x_{0}\right)=1$. Define

$$
D_{m}=\bigcup\left\{Q \in W \mid \frac{b^{-1}}{2^{m}} \leq d(Q) \leq \frac{b}{2^{m}}\right\}, \quad m=1,2, \ldots,
$$

where $b$ is chosen large enough so that $D=\cup D_{m}$, which is possible since $D$ is bounded, and so that the points $y_{i}$ satisfying (4.3) in Lemma 3 must belong to $D_{m_{i}}$. This can be done since $W$ is a Whitney decomposition. We also choose $b$ large enough so that $m\left(D_{m}\right)>0$ for all $m$.

Let $Q \in W$, then one easily sees that there is only a fixed number of sets $D_{m}$ that $Q$ can belong to and hence the function $\sum_{m} \chi_{D_{m}}$ is bounded on $D$. Here $\chi_{D_{m}}$ denotes the characteristic function of the set $D_{m}$. 
Let $x_{1} \in Q \in W$ and suppose that $Q \subset \bigcup_{3 n+n_{0}}^{\infty} D_{m}$. Since $\delta_{D}\left(x_{1}\right) \approx d(Q) \leq$ $b 2^{-3 n-n_{0}}$ we can choose $n_{0}$ so that $\delta_{D}\left(x_{1}\right) \leq 2^{-3 n}$. Hence by Lemma 3 we can find $n$ distinct integers $m_{1}, \ldots, m_{n}$ with $n \leq m_{i} \leq 3 n$ and points $y_{i} \in Q_{i} \in$ $W$ satisfying (4.3), where $Q_{i} \subset D_{m_{i}}$. Using the constants in (4.3) we see that there is a dilation $\tilde{Q}_{i}$ of $Q_{i}$, so that $x_{1} \in \tilde{Q}_{i}$ for all $i$, and $m\left(\tilde{Q}_{i}\right) \preceq m\left(Q_{i}\right)$. This means that

$$
1 \leq \frac{1}{n} \sum_{m=n}^{3 n} \chi_{\tilde{D}_{m}\left(x_{1}\right)}, \quad x_{1} \in \bigcup_{3 n+n_{0}} D_{m},
$$

where $\tilde{D}_{m}=\bigcup\left\{\tilde{Q} \mid Q \in W\right.$ and $\left.Q \subset D_{m}\right\}$.

Let $a_{m}=m\left(D_{m}\right)$ for $m \geq 0$. Using (4.6) and the fact that $\sum \chi_{D_{m}}$ is bounded we get

$$
\begin{aligned}
\sum_{3 n+n_{0}}^{\infty} a_{m} & =\int_{D} \sum_{3 n+n_{0}}^{\infty} \chi_{D_{m}} d x \preceq m\left(\bigcup_{3 n+n_{0}}^{\infty} D_{m}\right) \\
& \leq \int_{D} \frac{1}{n} \sum_{m=n}^{3 n} \chi_{\tilde{D}_{m}} d x \leq \frac{1}{n} \sum_{m=n}^{3 n} m\left(\tilde{D}_{m}\right) \\
& \preceq \frac{1}{n} \sum_{m=n}^{3 n} m\left(D_{m}\right)=\frac{1}{n} \sum_{m=n}^{3 n} a_{m} .
\end{aligned}
$$

Therefore, if we take $N=n \geq n_{0}$, then $3 n+n_{0} \leq 4 N$ and so

$$
\sum_{m=4 N}^{\infty} a_{m} \leq \sum_{m=3 n+n_{0}}^{\infty} a_{m} \leq \frac{c}{n} \sum_{m=n}^{3 n} a_{m} \leq \frac{c}{N} \sum_{m=N}^{4 N-1} a_{m} .
$$

Thus, the hypothesis to Lemma 2 is satisfied for large $N$. Since $a_{m}>0$ for all $m$ we can increase the constant in (4.1) so that it is true for all $N$. As in the proof of Theorem $4, k_{D}\left(x_{0}, x\right) \preceq m$ on $D_{m}$ and hence (1.7) follows from (4.2). This completes the proof of Theorem 2.

In a recent paper by $\mathrm{S}$. Staples, $L^{p}$-averaging domains are characterized by the $L^{p}$-integrability of $k_{D}\left(x_{0}, x\right)$. Hence Hölder domains are $L^{p}$-averaging domains, see Theorem 2.6 in [S]. Furthermore, Theorem 3.4 in that paper proves that $D$ is a $p$-Poincare domain provided that $k_{D}$ is in $L^{p}(D)$ and $p \geq d$. (See Theorem 9 below for a sharpening of this result.) Thus, the proof of Theorem 1 is completed and we also have the following corollary:

Corollary 3. If $D$ is a Hölder domain and $1 \leq p<\infty$, then

$$
\int_{D}\left|u-u_{D}\right|^{p} d x \leq c_{D} \sup _{B \subset D} \frac{1}{m(B)} \int_{B}\left|u-u_{B}\right|^{p} d x, \quad u \in L^{p}(D),
$$

where $c_{D}$ is a constant and the supremum is taken over all balls $B$ contained in $D$.

As a consequence of Lemma 3 we also have the following result. 
Corollary 4. If $D$ is a Hölder domain, then $m(\partial D)=0$.

Proof. Suppose that $m(\partial D)>0$. Let $y_{0} \in \partial D$ be a point of density for $\partial D$. This means that for any $\varepsilon>0$,

$$
\frac{m\left(B\left(y_{0}, r\right) \cap \partial D\right)}{m\left(B\left(y_{0}, r\right)\right)} \geq 1-\varepsilon
$$

provided $r$ is sufficiently small.

Let $x_{1} \in D \cap B\left(y_{0}, r\right)$. Assume that $x_{0} \notin B\left(y_{0}, r\right)$ and that $\left|x_{1}-y_{0}\right|$ is very small. By following a quasi-hyperbolic geodesic from $x_{1}$ to $x_{0}$, we can find a point $x \in D \cap B\left(y_{0}, r\right)$ with $\delta_{D}(x)$ comparable to $\left|x-y_{0}\right|$. Shrinking $r$ to $\left|x-y_{0}\right|$ results in a contradiction to $(4.8)$ provided $\varepsilon$ is sufficiently small. The proof is complete.

Remark. Domains are constructed in [S] where $k_{D}$ is in $L^{d-1}(D)$ and yet $m(\partial D)>0$.

Question. Does there exist a Hölder domain in $R^{d}$ whose boundary has Hausdorff dimension $d$ ?

\section{STARSHAPED DOMAINS IN $R^{d}$}

It is well known that balls are $p$-Poincaré domains and that $M_{p}(B(a, r)) \approx r$, for $1 \leq p<\infty$. Recall that $a \approx b$ means that $a / b$ is bounded from above and from below by positive dimensional constants. See Chapter 7 in [GT] for generalization to convex domains and Chapter 2 of $[\mathrm{M}]$ for domains which are starshaped with respect to an open set. In this section, we give new and simpler proofs of these theorems in addition to extending the generality to the class of bounded starshaped domains.

Lemma 4. If $Q=\left[0, a_{1}\right] \times \cdots \times\left[0, a_{d}\right]$, then $M_{p}(Q) \leq d \cdot \max \left(a_{1}, \ldots, a_{d}\right)$.

Proof. Assume that $\max \left(a_{1}, \ldots, a_{d}\right)=1$ and that $u \in C^{1}(Q)$. For $x, y \in Q$ and $1 \leq p<\infty$ we have that

$$
|u(x)-u(y)|^{p} \leq d^{p-1} \sum_{i=1}^{d} \int_{0}^{a_{i}}\left|\nabla u\left(y_{1}, \ldots, y_{i-1}, t, x_{i+1}, \ldots, x_{d}\right)\right|^{p} d t
$$

and hence that

$$
\begin{aligned}
\int_{Q} \mid u(x) & -\left.\frac{1}{m(Q)} \int_{Q} u(y) d y\right|^{p} d x \leq \frac{1}{m(Q)} \int_{Q} d x \int_{Q}|u(x)-u(y)|^{p} d y \\
& \leq d^{p-1} \sum_{i=1}^{d} \frac{1}{m(Q)} \int_{Q} \int_{Q} \int_{0}^{a_{i}}\left|\nabla u\left(y_{1}, \ldots, t, \ldots, x_{d}\right)\right|^{p} d t d x d y \\
& =d^{p-1} \sum_{i=1}^{d} a_{i} \int_{Q}|\nabla u|^{p} d x \leq d^{p} \int_{Q}|\nabla u|^{p} d x .
\end{aligned}
$$

The proof is now completed with a change of variables argument. 
Remark 1. In view of the Poincare inequality given on page 164 (page 157 in the first edition) in [GT] it is surprising that the Poincare constant, $M_{p}(Q)$, is independent of the small $a_{i}$ 's. Even for the unit cube $Q$, the constant in [GT] grows exponentially with $d$.

Remark 2. In general, one can modify the above argument to show that

$$
M_{p}\left(D_{1} \times D_{2}\right) \leq 3 \max \left(M_{p}\left(D_{1}\right), M_{p}\left(D_{2}\right)\right) .
$$

Denote by $Q(x, r)$ the cube with center $x$ and side length $2 r$.

Lemma 5. Suppose $Q(0,2 a) \subset D$ and $1 \leq p<\infty$. Let

$$
N_{p}(D)=\sup _{u}\left\{\frac{\|u\|_{L^{p}(D)}}{\|\nabla u\|_{L^{p}(D)}} \mid u \in C^{1}(D) \text { is nonconstant and } u=0 \text { on } B(0, a)\right\} \text {. }
$$

Then

$$
2^{-d-6} M_{p}(D) \leq N_{p}(D) \leq 2\left(1+\frac{m(D)}{m(B(0, a))}\right)^{1 / p} M_{p}(D) .
$$

Proof. Assume that $N_{p}(D)$ is finite. Let $u=0$ on $B(0, a), u=a$ off $B(0,2 a)$ and $u(x)=|x|-a$ at all other points. Then

$$
\frac{\|u\|_{L^{p}(D)}}{\|\nabla u\|_{L^{p}(D)}} \geq\left(\frac{\int_{a}^{2 a}(r-a)^{p} r^{d-1} d r}{\int_{a}^{2 a} r^{d-1} d r}\right)^{1 / p} \geq\left(\frac{a^{d+p} /(p+1)}{2^{d} a^{d} / d}\right)^{1 / p}
$$

and hence a limit argument yields the lower bound

$$
a\left(d / 2^{d}(p+1)\right)^{1 / p} \leq N_{p}(D) .
$$

Put $\varphi=1-u / a$ and suppose that $v$ is in $C^{1}(D)$ and satisfies $v_{Q(0,2 a)}=0$. Write $v=v_{1}+v_{2}$ where $v_{1}=\varphi v$. Since $v_{Q(0,2 a)}=0$ we have by Lemma 4 , that

$$
\|v\|_{L^{p}(Q(0,2 a))} \leq 4 a d\|\nabla v\|_{L^{p}(Q(0,2 a))} .
$$

Now $v_{2}=0$ on the set $B(0, a)$ and $|\nabla \varphi|=0$ off the set $B(0,2 a)$, thus it follows from (5.3) that

$$
\begin{aligned}
\left\|v_{2}\right\|_{L^{p}(D)} & \leq N_{p}(D)\left\|\nabla v_{2}\right\|_{L^{p}(D)} \\
& \leq N_{p}(D)\left(\|(1-\varphi) \nabla v\|_{L^{p}(D)}+\|v \nabla \varphi\|_{L^{p}(D)}\right) \\
& \leq N_{p}(D)\left(\|\nabla v\|_{L^{p}(D)}+\frac{1}{a}\|v\|_{L^{p}(Q(0,2 a))}\right) \\
& \leq 5 d N_{p}(D)\|\nabla v\|_{L^{p}(D)} .
\end{aligned}
$$

Finally, since $v_{1}=0$ off the set $Q(0,2 a)$ we use (5.3) again and (5.2) to obtain that

$$
\begin{aligned}
\left\|v-v_{D}\right\|_{L^{p}(D)} & \leq 2\left\|v-\left(v_{1}\right)_{D}\right\|_{L^{p}(D)} \\
& \leq 2\left(\left\|v_{1}-\left(v_{1}\right)_{D}\right\|_{L^{p}(D)}+\left\|v_{2}\right\|_{L^{p}(D)}\right)<10 d\left(a+N_{p}(D)\right)\|\nabla v\|_{L^{p}(D)} \\
& \leq 10 d\left(\left(2^{d}(p+1) / d\right)^{1 / p}+1\right) N_{p}(D)\|\nabla v\|_{L^{p}(D)} \\
& \leq 10\left(2^{d} e+d\right) N_{p}(D)\|\nabla v\|_{L^{p}(D)} \leq 2^{d+6} N_{p}(D)\|\nabla v\|_{L^{p}(D)} .
\end{aligned}
$$


This completes the proof of the first inequality in (5.1).

To prove the other inequaility in (5.1) notice first that if $u \in C^{1}(D)$ and $u_{B(0, a)}=0$, then

$$
\left|u_{D}\right|^{p} \leq\left(\frac{1}{m(B(0, a))} \int_{B(0, a)}\left|u-u_{D}\right| d x\right)^{1 / p} \leq \frac{1}{m(B(0, a))} \int_{D}\left|u-u_{D}\right|^{p} d x
$$

Thus

$$
\begin{aligned}
2^{-p} \int_{D}|u|^{p} d x & \leq \int_{D}\left|u-u_{D}\right|^{p} d x+\left|u_{D}\right|^{p} m(D) \\
& \leq\left(1+\frac{m(D)}{m(B(0, a))}\right) \int_{D}\left|u-u_{D}\right|^{p} d x \\
& \leq\left(1+\frac{m(D)}{m(B(0, a))}\right) M_{p}^{p}(D) \int_{D}|\nabla u|^{p} d x,
\end{aligned}
$$

and the result follows.

Theorem 5. If $Q(0,2 a) \subset D \subset B(0, b), 1 \leq p<\infty$ and $D$ is starshaped with respect to the origin, then

$$
M_{p}(D) \leq 2^{d+6} b\left(\frac{b}{a}\right)^{\frac{d-1}{p}} .
$$

Proof. Let $u \in C^{1}(D)$ and assume that $u=0$ on $B(0, a)$. For $\left|x^{\prime}\right|=1$ we assume that $r x^{\prime} \in D$ for $0 \leq r<b\left(x^{\prime}\right)$ and that $b\left(x^{\prime}\right) x^{\prime} \in \partial D$. By hypothesis, $2 a \leq b\left(x^{\prime}\right) \leq b$. For such $x^{\prime}$ we have the inequality

$$
\begin{aligned}
\int_{0}^{b\left(x^{\prime}\right)}\left|u\left(r x^{\prime}\right)\right|^{p} r^{d-1} d r & \leq b^{p} \int_{0}^{b\left(x^{\prime}\right)}\left(\frac{1}{r} \int_{a}^{r}\left|\nabla u\left(t x^{\prime}\right)\right| d t\right)^{p} r^{d-1} d r \\
& \leq b^{p} \int_{0}^{b} r^{d-2} d r \int_{a}^{b\left(x^{\prime}\right)}\left|\nabla u\left(t x^{\prime}\right)\right|^{p} d t \\
& \leq b^{p}\left(\frac{b}{a}\right)^{d-1} \int_{0}^{b\left(x^{\prime}\right)}\left|\nabla u\left(t x^{\prime}\right)\right|^{p} t^{d-1} d t
\end{aligned}
$$

and hence integration over the sphere yields that

$$
N_{p}(D) \leq b\left(\frac{b}{a}\right)^{\frac{d-1}{p}} .
$$

By Lemma 5 the result follows and the proof is complete.

For $1<p<\infty$, this last theorem can be improved by using Muckenhoupt's weighted norm inequality for the Hardy-Littlewood maximal function as we did earlier for the analytic Poincare inequality in the plane, see Theorem 2.1 in [SS2].

Theorem 6. If $Q(0,2 a) \subset D \subset B(0, b)$ and $1 \leq p<\infty$ and $D$ is starshaped with respect to the origin, then there are constants $c_{p}=c_{p}(p, d)$ such that

$$
M_{p}^{p}(D) \leq c_{p} b^{p} \begin{cases}\left(\frac{b}{a}\right)^{d-p}, & 1 \leq p<d, \\ \left(\log \left(\frac{b}{a}+1\right)\right)^{d-1}, & p=d, \\ 1, & p>d .\end{cases}
$$


Proof. The case $p=1$ is contained in Theorem 5. Fix $1<p<\infty$ and define $w(t)=\min \left((a+|t|)^{d-1}, b^{d-1}\right)$ for $-\infty<t<\infty$. Then a straightforward calculation shows that

$$
A_{p}=\sup _{I} \frac{1}{|I|} \int_{I} w d t\left(\frac{1}{|I|} \int_{I} w^{-\frac{1}{p-1}} d t\right)^{p-1}
$$

is comparable to the quantities on the right hand side of (5.6).

We modify the proof of Theorem 5 slightly to obtain that whenever $\left|x^{\prime}\right|=1$ and $u=0$ on $B(0, a)$, we have that

$$
\begin{aligned}
\int_{0}^{b\left(x^{\prime}\right)}\left|u\left(r x^{\prime}\right)\right|^{p} r^{d-1} d r & \leq b^{p} \int_{a}^{b\left(x^{\prime}\right)}\left(\frac{1}{r-a} \int_{a}^{r}\left|\nabla u\left(t x^{\prime}\right)\right| d t\right)^{p} r^{d-1} d r \\
& \leq c_{p} b^{p} A_{p} \int_{0}^{b\left(x^{\prime}\right)}\left|\nabla u\left(r x^{\prime}\right)\right|^{p} r^{d-1} d r
\end{aligned}
$$

where this last inequality is due to Muckenhoupt, see [Mu]. The proof is then completed as previously.

The constants on the right-hand side of (5.6) can be improved if the domain $D$ is such that integration with respect to polar coordinates in the proof can be replaced by integration with respect to rectangular coordinates. For $p>1$, this is because the weight $w(t)$ in (5.7) reduces to $w(t)=1$, so that $A_{p}=1$. A similar modification of the proof of Theorem 5 works for $p=1$. Using these ideas we obtain the following theorem; we omit further details. It generalizes Theorem 2.2 of [SS2], and will be required in section 10 . We use $Q^{d-1}$ to denote a cube in $R^{d-1}$.

Theorem 7. Suppose that $h(x)$ is a lower semicontinuous function defined on $Q^{d-1}(0, a)$ and satisfies $0<a \leq h(x) \leq b$ for all $x$. If

$$
D=B(0,2 a) \cup\left\{(x, t): x \in Q^{d-1}(0, a),-a<t<h(x)\right\},
$$

then $M_{p}^{p}(D) \leq c_{p} b^{p}, 1 \leq p<\infty$.

\section{A SUFFICIENT CONDITION FOR THE POINCARÉ INEQUALITY}

We assume throughout that $D$ is a domain in $R^{d}$ with finite volume and Whitney decomposition $W$. Fix a cube $Q_{0} \in W$ and let $x_{0}$ be its center. We assume that, for each $Q \in W$, there is a set $P(Q) \subset D$ containing a chain $Q_{0}, \ldots, Q_{n}$ of cubes in $W$, starting with $Q_{0}$ and ending with $Q_{n}=Q$. This means that $Q_{i}$ is adjacent to $Q_{i+1}$ in $W$, so that a face of the smaller cube is contained in a face of the larger cube, and $Q_{i} \subset P(Q)$ for $0 \leq i \leq n$. Now define

$$
S(Q)=\bigcup\left\{Q_{1} \in W \mid Q \subset P\left(Q_{1}\right)\right\} .
$$

As an example of how one might construct the the sets $P(Q)$, we could simply take the cubes in $W$ which intersect a quasi-hyperbolic geodesic joining 
$x_{0}$ to the center of $Q$. A geometric interpretation of $S(Q)$, in this case, is that it is the points in the shadow of $Q$, assuming that light travels from $x_{0}$ along the quasi-hyperbolic geodesics in $D$. For $x \in D$, let $Q_{x}$ denote a cube in $W$ containing $x$.

Theorem 8. If $D$ satisfies the above conditions and $\lambda$ is a real number, then

$$
M_{p}(D) \preceq \sup _{Q \in W}\left\{\int_{S(Q)}\left(\int_{P\left(Q_{x}\right)} \frac{d y}{\delta_{D}(y)^{p^{\prime}(1-\lambda)(d-1)}}\right)^{p-1} \frac{d x}{d(Q)^{\lambda p(d-1)}}\right\}^{1 / p}
$$

for $1<p<\infty, p^{\prime}=\frac{p}{p-1}$ and

$$
M_{1}(D) \preceq \sup _{Q \in W} \frac{m(S(Q))}{d(Q)^{d-1}}
$$

when $p=1$.

Remark. Although we have stated Theorem 8 for an arbitrary real number $\lambda$, the result is only of interest for $\lambda$ satisfying $\frac{d-1-p}{p(d-1)}<\lambda \leq \frac{d}{p(d-1)}$. The right side of (6.2) will be infinite for any other choice of $\lambda$. We omit the details of this computation.

Lemma 6. Let $Q_{1}, Q_{2}$ be adjacent cubes in $W$. Then $M_{p}\left(Q_{1}\right)$ and $M_{p}\left(Q_{1} \cup Q_{2}\right)$ are both comparable to $d\left(Q_{1}\right)$, for $1 \leq p<\infty$.

Proof. The first assertion is Lemma 4 and the second follows from Theorem 5.

Remark. Lemma 6 is well known and follows from the uniform cone condition (see $\S 1.1 .11$ of $[\mathrm{M}]$ ). We give a new proof of this classical result in Theorem 10 .

Lemma 7. For $1 \leq p<\infty$,

$$
M_{p}(D) \preceq \sup _{Q \in W} d(Q)+\sup _{u} \frac{\left(\sum_{Q \in W}\left|u_{Q}-u_{Q_{0}}\right|^{p} m(Q)\right)^{1 / p}}{\|\nabla u\|_{L^{p}(D)}} .
$$

Proof. For $u \in C^{1}(D)$, we have that

$$
\begin{aligned}
\int_{D}\left|u-u_{D}\right|^{p} d x & \leq 2^{p} \int_{D}\left|u-u_{Q_{0}}\right|^{p} d x=2^{p} \sum_{Q \in W} \int_{Q}\left|u-u_{Q_{0}}\right|^{p} d x \\
& \leq 4^{p} \sum_{Q \in W}\left(\int_{Q}\left|u-u_{Q}\right|^{p} d x+\left|u_{Q}-u_{Q_{0}}\right|^{p} m(Q)\right) \\
& \leq 4^{p}\left(\sup _{Q \in W} M_{p}^{p}(Q) \int_{D}|\nabla u|^{p} d x+\sum_{Q \in W}\left|u_{Q}-u_{Q_{0}}\right|^{p} m(Q)\right) .
\end{aligned}
$$

The result now follows from Lemma 6.

Lemma 8. If $u \in C^{1}(D)$ and $Q \in W$, then

$$
\left|u_{Q}-u_{Q_{0}}\right| \preceq \int_{P(Q)} \frac{|\nabla u|}{\delta_{D}(x)^{d-1}} d x
$$


Proof. Let $Q_{0}, \ldots, Q_{n}$ be a minimal chain of cubes in $P(Q)$ which joins $Q_{0}$ to $Q$. The $Q_{i}$ 's must then be distinct, for if this were not the case, we could remove a subsequence of adjacent cubes and still have a chain from $Q_{0}$ to $Q$. Using Lemma 6 we then have that

$$
\begin{aligned}
\left|u_{Q}-u_{Q_{0}}\right| & \leq \sum_{i=1}^{n}\left|u_{Q_{i}}-u_{Q_{i-1}}\right| \\
& \leq \sum_{i=1}^{n} \frac{1}{m\left(Q_{i} \cup Q_{i-1}\right)} \int_{Q_{i} \cup Q_{i-1}}\left|u-u_{Q_{i} \cup Q_{i-1}}\right| d x \\
& \leq \sum_{i=1}^{n} \frac{M_{1}\left(Q_{i} \cup Q_{i-1}\right)}{m\left(Q_{i} \cup Q_{i-1}\right)} \int_{Q_{i} \cup Q_{i-1}}|\nabla u| d x \\
& \preceq \sum_{i=1}^{n} \frac{1}{d\left(Q_{i}\right)^{d-1}} \int_{Q_{i} \cup Q_{i-1}}|\nabla u| d x \\
& \preceq \int_{\bigcup Q_{i}} \frac{|\nabla u|}{\delta_{D}(x)^{d-1}} d x \leq \int_{P(D)} \frac{|\nabla u|}{\delta_{D}(x)^{d-1}} d x
\end{aligned}
$$

and the proof is complete.

Proof of Theorem 8. Let $M$ denote the right-hand side of (6.2) and assume that it is finite. If $Q \in W$, then $Q \subset S(Q) \cap P(Q)$ and hence for some $\lambda$ we have that

and it follows that

$$
\frac{m(Q)}{d(Q)^{\lambda p(d-1)}}\left(\int_{Q} \frac{d y}{\delta_{D}(y)^{p^{\prime}(1-\lambda)(d-1)}}\right)^{p-1} \leq M^{p}
$$

$$
d(Q) \preceq M, \quad Q \in W .
$$

Suppose that $u \in C_{1}(D)$ and put

$$
F_{p}(x, \lambda)=\left(\int_{P\left(Q_{x}\right)} \frac{d y}{\delta_{D}(y)^{p^{\prime}(1-\lambda)(d-1)}}\right)^{p-1}
$$

for $x \in D,-\infty<\lambda<\infty$ and $1<p<\infty$. For $p=1$, let $F_{1}=1$ and set $\lambda=1$. Using Lemma 8 and Hölder's inequality we see that

$$
\begin{aligned}
\sum_{Q \in W}\left|u_{Q}-u_{Q_{0}}\right|^{p} m(Q) & \leq \sum_{Q \in W}\left(\int_{P(Q)} \frac{|\nabla u| d x}{\delta_{D}(x)^{d-1}}\right)^{p} m(Q) \\
& \leq \sum_{Q \in W} \int_{P(Q)} \frac{|\nabla u|^{p} d x}{\delta_{D}(x)^{\lambda p(d-1)}} F_{p}\left(x_{Q}, \lambda\right) m(Q) \\
& =\int_{D}|\nabla u|^{p}\left\{\sum_{Q \in W} \frac{\chi_{P(Q)}(x) F_{p}\left(x_{Q}, \lambda\right)}{\delta_{D}(x)^{\lambda p(d-1)}} m(Q)\right\} d x .
\end{aligned}
$$

Since $m(\bigcup\{\partial Q \mid Q \in W\})=0$, we need only consider $x \in D$ with $x$ in the interior of some $Q_{1} \in W$. For such $x, \chi_{P(Q)}(x) \neq 0$ if and only if $Q \subset S\left(Q_{1}\right)$. 
In addition, $d\left(Q_{1}\right) \leq c \delta_{D}(x)$, for $x \in Q_{1}$, where $c \approx 1$. Thus, we simplify the above to get that

$$
\begin{aligned}
\sum_{Q \in W}\left|u_{Q}-u_{Q_{0}}\right|^{p} m(Q) & \leq c^{\lambda p(d-1)} \int_{D}|\nabla u|^{p}\left(\int_{S\left(Q_{x}\right)} F_{p}(y, \lambda) \frac{d y}{d\left(Q_{x}\right)^{\lambda p(d-1)}}\right) d x \\
& \leq c^{\lambda p(d-1)} M^{p} \int_{D}|\nabla u|^{p} d x
\end{aligned}
$$

and hence that

$$
\frac{\left(\sum_{Q \in W} \mid u_{Q}-u_{Q_{0}}{ }^{p} m(Q)\right)^{1 / p}}{\|\nabla u\|_{L^{p}(D)}} \preceq M
$$

Finally, Lemma 7 combined with (6.6) and (6.8) give the required bound on $M_{p}(D)$ and the proof is complete.

\section{JOHN DOMAINS WITH CUSPS AND INTEGRABILITY CONDITIONS FOR $K_{D}$}

We give some applications of Theorem 8 in this section by constructing sets $P(Q)$ from families of arcs in $D$ generated by a new distance function on $D$. For $1<p<\infty$ the metric $k_{p}$ in $D$ is defined by

$$
k_{p}\left(x_{1}, x_{2}\right)=\inf _{\gamma} \int_{\gamma} \frac{d s}{\delta_{D}(x)^{\frac{d-1}{p-1}}}
$$

where the infimum is taken over all rectifiable arcs $\gamma$ joining $x_{1}$ to $x_{2}$ in $D$. Notice that $k_{d}=k_{D}$. Martin has shown that geodesics exist for $k_{p}$ if $1<p \leq d$ [Ma, 2.11], but we will not need this fact.

Theorem 9. For $1 \leq p<\infty$,

$$
M_{p}(D) \preceq \begin{cases}\sup _{Q \in W}\left(\frac{1}{d(Q)^{d-p}} \int_{S(Q)} k_{D}^{p-1}\left(x_{0}, x\right) d x\right)^{1 / p}, & 1 \leq p<d, \\ \left(\int_{D} k_{D}^{p-\frac{p}{d}}\left(x_{0}, x\right) d x \cdot m(D)^{r}\right)^{1 / p}, & d \leq p,\end{cases}
$$

where $r=\frac{p-d}{d(p-1)}$. For $d-1<p<\infty$,

$$
M_{p}(D) \preceq\left(\int_{D} k_{p}^{p-1}\left(x_{0}, x\right) d x\right)^{1 / p} .
$$

Remarks. The statement of Theorem 9 has $p>d-1$ in (7.2) since otherwise the right-hand side is infinite, as is easily checked.

For $d \leq p$

$$
k_{p}^{p-1}\left(x_{0}, x\right) \preceq k_{D}^{p-\frac{p}{d}}\left(x_{0}, x\right) \cdot m(D)^{r}
$$

where $r$ is as in Theorem 9. This follows from the application of Hölder's inequality in (7.5) below. Thus (7.2) is stronger than (7.1) if $p \geq d$. 
Proof. Let $W$ be a Whitney decomposition of $D$ and for each $Q \in W$, let $\gamma_{Q}$ be a fixed arc joining $x_{0}$ to the center of $Q$, such that

$$
\int_{\gamma_{Q}} \frac{d s}{\delta_{D}(x)^{\frac{d-1}{p-1}}} \leq 2 k_{p}\left(x_{0}, x_{Q}\right)
$$

Define $P(Q)$ to be the union over the cubes in $W$ which intersect $\gamma_{Q}$.

Lemma 9. For $1<p<\infty$,

$$
\int_{P(Q)} \frac{d y}{\delta_{D}(y)^{p^{\prime}(d-1)}} \preceq k_{p}\left(x_{0}, x\right)
$$

whenever $x \in Q, Q \in W$ and $Q \neq Q_{0}$.

Proof. Fix $x \in Q$ with $Q \in W$ and $Q \neq Q_{0}$. By the triangle inequality, $k_{p}\left(x_{0}, x_{Q}\right) \leq k_{p}\left(x_{0}, x\right)+k_{p}\left(x, x_{Q}\right)$. Since $W$ is a Whitney decomposition it is obvious from the definition that $k_{p}\left(x, x_{Q}\right) \preceq d(Q)^{1-(d-1) /(p-1)}$. Let $\gamma$ be an arc in $D$ joining $x_{0}$ to $x$. Since $Q \neq Q_{0}$ ]we can find a cube $Q_{1} \in W$ with $d\left(Q_{1}\right) \approx d(Q)$ and $d\left(Q_{1}\right) \preceq\left|\gamma \cap Q_{1}\right|$. Hence

$$
\int_{\gamma} \frac{d s}{\delta_{D}(y)^{\frac{d-1}{p-1}}} \succeq \frac{\left|\gamma \cap Q_{1}\right|}{d\left(Q_{1}\right)^{\frac{d-1}{p-1}}} \succeq d(Q)^{1-\frac{d-1}{p-1}} \succeq k_{p}\left(x, x_{Q}\right) .
$$

Thus, $k_{p}\left(x_{0}, x\right) \succeq k_{p}\left(x, x_{Q}\right)$ and so $k_{p}\left(x_{0}, x_{Q}\right) \preceq k_{p}\left(x_{0}, x\right)$ whenever $x \in Q$. Hence it suffices to prove (7.4) with $x=x_{Q}$.

Let $\gamma_{Q}$ be the arc in (7.3). For $R \in W$, denote by $\tilde{R}$, the union of all cubes in $W$ which have a nonempty intersection with $R$. It is easily seen that there are cubes $Q_{1}, \ldots, Q_{k}$ in $W$ for which (a) $\gamma_{Q} \cap Q_{i}$ is nonempty for $1 \leq i \leq k$, (b) $Q_{i} \not \subset \tilde{Q}_{j}$ for $i \neq j$ and (c) $P(Q) \subset \cup \tilde{Q}_{i}$. Clearly, we then have that

$$
\begin{aligned}
\int_{P(Q)} \frac{d y}{\delta_{D}(y)^{p^{\prime}(d-1)}} & \leq \sum_{i=1}^{k} \int_{\dot{Q}_{i}} \frac{d y}{\delta_{D}(y)^{p^{\prime}(d-1)}} \\
& \approx \sum_{i=1}^{k} d\left(Q_{i}\right)^{d-p^{\prime}(d-1)}=\sum_{i=1}^{k} d\left(Q_{i}\right)^{1-\frac{d-1}{p-1}} \\
& \leq \sum_{i=1}^{k} \int_{\gamma_{Q} \cap \dot{Q}_{i}} \frac{d s}{\delta_{D}(y)^{\frac{d-1}{p-1}}} \\
& \preceq \int_{\gamma_{Q}} \frac{d s}{\delta_{D}(y)^{\frac{d-1}{p-1}}} \leq 2 \cdot k_{p}\left(x_{0}, x_{Q}\right) .
\end{aligned}
$$

This completes the proof of Lemma 9. 
Returning to the proof of Theorem 9 , we let $d-1<p<\infty$, apply Theorem 8 with $\lambda=0$ and use Lemma 9 to conclude (7.2):

$$
\begin{aligned}
M_{p}(D) & \preceq\left(\int_{D}\left(\int_{P\left(Q_{x}\right)} \frac{d y}{\delta_{D}(y)^{p^{\prime}(d-1)}}\right)^{p-1} d x\right)^{1 / p} \\
& \preceq\left(\int_{D} k_{p}^{p-1}\left(x_{0}, x\right) d x\right)^{1 / p} .
\end{aligned}
$$

For $p \geq d$ we have that $p^{\prime}(d-1) \leq d$. Arguing in a similar manner, but using the Hölder inequality we get that

$$
\begin{aligned}
M_{p}(D) & \preceq\left(\int_{D}\left(\int_{P\left(Q_{x}\right)} \frac{d y}{\delta_{D}(y)^{p^{\prime}(d-1)}}\right)^{p-1} d x\right)^{1 / p} \\
& \leq\left(\int_{D}\left(\int_{P\left(Q_{x}\right)} \frac{d y}{\delta_{D}(y)^{d}}\right)^{p-\frac{p}{d}} m\left(P\left(Q_{x}\right)\right)^{r} d x\right)^{1 / p} \\
& \preceq\left(\int_{D} k_{D}^{p-\frac{p}{d}}\left(x_{0}, x\right) d x \cdot m(D)^{r}\right)^{1 / p},
\end{aligned}
$$

and the proof of $(7.1)$ is complete in this case.

Observe that Lemma 9 , with $p=d$, was used in this last argument. We choose to use quasi-hyperbolic geodesics $\gamma_{Q}$ in this case, to generate the sets $P(Q)$.

Finally, for the case $1<p<d$ in (7.1) we apply Theorem 8 with $\lambda=$ $(d-p) / p(d-1)$ to obtain the inequality

$$
\begin{aligned}
M_{p}(D) & \preceq \sup _{Q \in W}\left(\frac{1}{d(Q)^{d-p}} \int_{S(Q)}\left(\int_{P\left(Q_{x}\right)} \frac{d y}{\delta_{D}^{d}(y)}\right)^{p-1} d x\right)^{1 / p} \\
& \preceq \sup _{Q \in W}\left(\frac{1}{d(Q)^{d-p}} \int_{S(Q)} k_{D}^{p-1}\left(x_{0}, x\right) d x\right)^{1 / p},
\end{aligned}
$$

which proves (7.1) for $1<p<d$. The case $p=1$ is part of Theorem 8 and thus the proof of Theorem 9 is complete.

Definition. Let $D$ be a domain and $x_{0} \in D$. We say that $D$ is an $\eta$-John domain for $\eta \geq 1$ provided there is a constant $\alpha>0$ such that, for each $x_{1} \in D$, there is an arc $\gamma$ joining $x_{0}$ to $x_{1}$ in $D$ along which

$$
\delta_{D}(x) \geq \alpha\left|\gamma\left(x, x_{1}\right)\right|^{\eta}, \quad x \in \gamma .
$$

Notice that when $\eta=1$ this definition agrees with the definition of John domains given in the introduction. Power cusps are allowed in $\eta$-John domains for $\eta>1$, and so these domains comprise a larger class of domains than John domains. Martio has shown that a John domain is a $p$-Poincaré domain for all $p \geq 1$ [Mar]. We now give an extension of this result to $\eta$-John domains. 
Theorem 10. Let $1 \leq p<\infty$. If $D$ is an $\eta$-John domain with

$$
\eta<\frac{d}{d-1}+\frac{p-1}{d-1}
$$

then $D$ is a p-Poincaré domain. In particular, if $\eta<\frac{d}{d-1}$, then $D$ is a p-Poincaré domain for all $1 \leq p<\infty$.

Proof. Let $W$ be a Whitney decomposition for $D$. For each $Q \in W$, let $\gamma$ be an arc joining $x_{0}$ to $x_{Q}$ along which (7.6) holds. For each pair of adjacent cubes in $W$ we fix an arc joining their centers which is contained in their union and whose length is comparable to their side lengths. We replace replace $\gamma$ by an arc $\gamma_{Q}$ constructed from these special arcs. This can be done so as to still satisfy (7.6) provided we account for an increase of the arc length by a constant factor. We therefore have that

$$
\begin{aligned}
& \delta_{D}(x) \geq \frac{\alpha}{c^{\eta}}\left|\gamma_{Q}\left(x, x_{Q}\right)\right|^{\eta}, \quad x \in \gamma_{Q}, \\
& d\left(Q_{1}\right) \preceq\left|\gamma_{Q} \cap Q_{1}\right|, \quad Q_{1} \cap \gamma_{Q} \neq \varnothing, \quad Q_{1} \in W,
\end{aligned}
$$

where $c$ is a dimensional constant. Let $P(Q)$ be the union of the cubes in $W$ which intersect $\gamma_{Q}$.

Let $0<\lambda \leftrightharpoons 1$ be determined by

$$
\lambda p=\frac{d}{d+p-1} \leq 1
$$

Using the property that $d\left(Q_{1}\right) \preceq\left|\gamma_{Q} \cap Q_{1}\right|$ for each $Q_{1}$ in $P(Q)$ we can replace volume measure $d y$ on $Q_{1}$ by $\delta_{D}^{d-1}(y) d s$ on $\gamma_{Q} \cap Q_{1}$ and hence we get that

$$
\left(\int_{P(Q)} \frac{d y}{\delta_{D}(y)^{p^{\prime}(1-\lambda)(d-1)}}\right)^{p-1} \preceq\left(\int_{\gamma_{Q}} \frac{d s}{\delta_{D}(y)^{\frac{(1-p \lambda)(d-1)}{p-1}}}\right)^{p-1} .
$$

Let $L=c\left(\delta_{D}\left(x_{0}\right) / \alpha\right)^{1 / \eta}$, then from (7.8) it follows that $\gamma_{Q} \leq L$. If we use euclidean distance as a lower bound for arc length and use that (7.9) implies that $\eta(1-p \lambda)(d-1)<p-1$ we obtain that

$$
\left(\int_{\gamma_{Q}} \frac{d s}{\delta_{D}(y)^{\frac{(1-p \lambda)(d-1)}{p-1}}}\right)^{p-1} \leq\left(\frac{c^{\eta}}{\alpha}\right)^{(1-p \lambda)(d-1)}\left(\int_{0}^{L} \frac{d s}{s^{\frac{\eta(1-p \lambda)(d-1)}{p-1}}}\right)^{p-1}=A<\infty
$$

and hence the right-hand side of (7.10) is bounded by a constant independent of $Q$.

In a similar manner we obtain an upper bound for $m(S(Q))$. If $x$ is in the interior of $Q_{1} \subset S(Q)$, then the arc $\gamma_{Q_{1}}$ passes through the center of $Q$. By (7.8) we have that

$$
d(Q) \geq \frac{\alpha}{c_{1}^{\eta}}\left|x-x_{Q}\right|^{\eta}
$$


for a constant $c_{1}$ and therefore

$$
\begin{aligned}
\frac{m(S(Q))}{d(Q)^{\lambda p(d-1)}} & \leq \frac{c_{1}^{\eta \lambda p(d-1)}}{\alpha^{\lambda p(d-1)}} \int_{S(Q)} \frac{d x}{\left|x-x_{Q}\right|^{\eta \lambda p(d-1)}} \\
& \leq \frac{c_{1}^{\eta \lambda p(d-1)}}{\alpha^{\lambda p(d-1)}} \int_{B(0, L)} \frac{d x}{|x|^{\eta \lambda p(d-1)}}=B<\infty
\end{aligned}
$$

since (7.7) implies that $\eta \lambda p(d-1)<d$.

Finally, by combining (7.10), (7.11) and the above we have that

$$
\sup _{Q} \int_{S(Q)}\left(\int_{P(Q)} \frac{d y}{\delta_{D}(y)^{p^{\prime}(1-\lambda)(d-1)}}\right)^{p-1} \frac{d x}{d(Q)^{\lambda p(d-1)}} \leq A \cdot B<\infty
$$

and the proof is completed by applying Theorem 8 .

\section{Compactness of THE IMBEDding $W^{1, p}(D) \rightarrow L^{p}(D)$}

In this section we are concerned with the question of when the imbedding of a Sobolev space into $L^{p}$ is compact and the implications this has for the Poincaré inequality. Questions of compactness are important for applications, in part because compact operators have discrete spectra. The study of compact imbeddings began with Rellich [R]. See also Chapter 6 of [Ad].

For a domain $D$ in $R^{d}$ and $1 \leq p<\infty$, recall that $W^{1, p}(D)$ is the usual Sobolev space of functions on $D$ (see the introduction). Obviously, the natural imbedding of $W^{1, p}(D)$ into $L^{p}(D)$ is continuous. The next theorem gives an equivalent condition for the imbedding to be compact. Amick considered a closely related condition and the question of compactness for a bounded domain with $p=2$ in $[\mathrm{Am}]$.

Theorem 11. Let $1 \leq p<\infty$ and let $D$ be an open set in $R^{d}$. The imbedding $W^{1, p}(D) \rightarrow L^{p}(D)$ is compact if and only if

$$
\lim _{n \rightarrow \infty} \sup \left\{\int_{D \backslash D_{n}}|u|^{p} d x \mid\|u\|_{W^{1, p}(D)} \leq 1\right\}=0,
$$

where $D_{n}=\left\{x \in D \mid \delta_{D}(x)>1 / n\right.$ and $\left.|x|<n\right\}$.

This theorem is an easy consequence of the following lemma. We follow the convention of extending $u \in L^{p}(D)$ to be defined in $R^{d}$ by setting $u$ equal to 0 on $R^{d} \backslash D$.

Lemma 10 [Ad, 2.21, p. 31]. Let $1 \leq p<\infty$. A bounded subset $X$ in $L^{p}(D)$ is precompact in $L^{p}(D)$ if and only if for every $\varepsilon>0$ there exists $\delta>0$ and a compact subset $K$ of $D$ such that

$$
\int_{D}|u(x+h)-u(x)|^{p} d x<\varepsilon^{p}, \quad|h|<\delta, u \in X,
$$


and

$$
\int_{D \backslash K}|u|^{p} d x<\varepsilon^{p}, \quad u \in X
$$

Proof of Theorem 11. First suppose that the imbedding is compact. We apply Lemma 10 with $X=\left\{u \in W^{1, p}(D) \mid\|u\|_{W^{1, p}(D)} \leq 1\right\}$. Thus, given $\varepsilon>0$ there is a compact set $K \subset D$ such that (8.3) holds. Since $K \subset D_{n}$ for all sufficiently large $n,(8.1)$ follows.

For the converse, we adapt an argument from [Ad, p. 147]. Suppose that (8.1) holds. Let $X=C^{1}(D) \cap\left\{u \in W^{1, p}(D) \mid\|u\|_{W^{1, p}(D)} \leq 1\right\}$. Then if $u \in X$ and $h<1 / n$, we have

$$
\begin{aligned}
\int_{D_{n}}|u(x+h)-u(x)|^{p} d x & \leq \int_{D_{n}}\left(\int_{0}^{1}\left|\frac{d}{d t} u(x+t h)\right| d t\right)^{p} d x \\
& \leq|h|^{p} \int_{D_{n}} d x \int_{0}^{1}|\nabla u(x+t h)|^{p} d t \\
& \leq|h|^{p} \int_{D}|\nabla u|^{p} d x .
\end{aligned}
$$

This fact together with (8.1) clearly shows that (8.2) holds for $u \in X$. Furthermore, (8.3) follows immediately from (8.1) by letting $K$ be the closure of $D_{n}$ for $n$ sufficiently large. The compactness of the imbedding now follows from the Lemma 10, and the proof is complete.

Corollary 5. Let $d-1<p<\infty$ and $x_{0} \in D$. If

$$
\int_{D} k_{p}^{p-1}\left(x_{0}, x\right) d x<\infty
$$

then the imbedding $W^{1, p}(D) \rightarrow L^{p}(D)$ is compact.

Proof. Recall that $d-1<p$ is necessary for (8.4) to hold. Let $W$ be a Whitney decomposition of $D$ and let $\left\{D_{n}\right\}$ be as in (8.1). Then there are positive constants $c_{1}$ and $c_{2}$ such that

$$
D \backslash D_{n} \subset \bigcup\left\{Q \in W \mid d(Q) \leq c_{1} / n\right\} \subset D \backslash D_{n c_{2}}, \quad n \geq 1
$$

Suppose that $\|u\|_{W^{1, p}(D)} \leq 1$. We then have

$$
\begin{aligned}
\int_{D \backslash D_{n}}|u|^{p} d x & \leq \sum_{d(Q) \leq c_{1} / n} \int_{Q}|u|^{p} d x \\
\leq & 3^{p} \sum_{d(Q) \leq c_{1} / n}\left\{\int_{Q}\left|u-u_{Q}\right|^{p} d x+\left|u_{Q}-u_{Q_{0}}\right|^{p} m(Q)\right\} \\
& +3^{p}\left|u_{Q_{0}}\right|^{p} m\left(D \backslash D_{n c_{2}}\right) .
\end{aligned}
$$

Now,

$$
\sum_{d(Q) \leq c_{1} / n} \int_{Q}\left|u-u_{Q}\right|^{p} d x \leq \sup _{d(Q) \leq c_{1} / n} M_{p}^{p}(Q) \int_{D \backslash D_{n c_{2}}}|\nabla u|^{p} d x \leq\left(\frac{c_{3}}{n}\right)^{p}
$$


where $c_{3}$ is a dimensional constant by Lemma 6 . Also, as in the proof of Theorem 8 (with $\lambda=0$ ) we get that

$$
\sum_{d(Q) \leq c_{1} / n}\left|u_{Q}-u_{Q_{0}}\right|^{p} m(Q) \preceq \int_{D}|\nabla u|^{p} d x \int_{D \backslash D_{n c_{2}}} k_{p}^{p-1}\left(x_{0}, x\right) d x=o(1),
$$

since $k_{p}\left(x_{0}, x\right) \in L^{p-1}(D)$. The last term on the right side of (8.5) also goes to zero as $n$ tends to infinity since $D$ clearly has finite volume by condition (8.4). So we have that (8.1) holds and we are done by Theorem 11 .

The following result is an immediate consequence of Theorem 2, Corollary 5 and the Remark in $\S 7$.

Corollary 6. If $p \geq d$ and $D$ is a Hölder domain, then the imbedding $W^{1, p}(D)$ $\rightarrow L^{p}(D)$ is compact.

We next consider the relationship between the Poincare inequality and the compactness of the imbedding $W^{1, p}(D) \rightarrow L^{p}(D)$. The following result may well be known. We include a proof since we have been unable to find a reference.

Theorem 12. Suppose that $1 \leq p<\infty$ and $m(D)<\infty$. If the imbedding $W^{1, p}(D) \rightarrow L^{p}(D)$ is compact, then $M_{p}(D)<\infty$.

Proof. Arguing by contradiction we assume that the imbedding is compact but that $M_{p}(D)$ is infinite. Then there is a sequence $\left\{u_{n}\right\} \subset C^{1}(D)$ such that

$$
\int_{D}\left|u_{n}\right|^{p} d x=1, \quad \int_{D} u_{n} d x=0 \text { and } \lim _{n \rightarrow \infty} \int_{D}\left|\nabla u_{n}\right|^{p} d x=0 .
$$

By the compactness of the imbedding there is a subsequence which we continue to denote $\left\{u_{n}\right\}$ and $u \in L^{p}(D)$ such that $u_{n} \rightarrow u$ in $L^{p}(D)$. Now let $\alpha$ be a multi-index with $|\alpha|=1$ and let $\phi \in C_{0}^{\infty}(D)$, where this denotes functions compactly supported on $D$ and having continuous partial derivatives of all orders. Then

$$
D^{\alpha} u(\varphi)=-\int_{D} u D^{\alpha} \varphi d x=-\lim _{n \rightarrow \infty} \int_{D} u_{n} D^{\alpha} \varphi d x=\lim _{n \rightarrow \infty} \int_{D} \varphi D^{\alpha} u_{n} d x=0
$$

since $\left\|\nabla u_{n}\right\|_{L^{p}(D)} \rightarrow 0$ by (8.6). Thus $u$ has a weak gradient in $D$ and $\nabla u=0$. Since $M_{p}(B)<\infty$ for each ball $B \subset D$, we see that this means that $u$ is locally constant in $D$. Since $D$ is a connected set, $u$ is almost everywhere equal to a constant in $D$. This is a contradiction since it follows from (8.6) that $\|u\|_{L^{p}(D)}=1$ and $\int_{D} u d x=0$, and the proof is complete.

\section{EXAMPLE OF A NONCOMPACT EMBEDDING}

In $\S 8$ we showed that the sufficient condition for the Poincare inequality from Theorem 10 actually implies that the imbedding $W^{1, p}(D) \rightarrow L^{p}(D)$ is compact. We now show that this does not extend to all of our other sufficient conditions for the Poincaré inequality. More precisely, we construct a domain 
$D$ for which the imbedding $W^{1, p}(D) \rightarrow L^{p}(D)$ is not compact, and we use (6.2) of Theorem 8 with $p=2, d=3$ and $\lambda=1 / 4$ to show $M_{2}(D)<\infty$.

Let $\left\{x_{i}\right\}_{i=1}^{\infty}$ be a sequence of points in $R^{3}$ such that $\left|x_{i}\right|=1$ and the balls $\left\{B\left(x_{i}, 2^{-i}\right)\right\}$ are pairwise disjoint. Now set $y_{i}=\left(1+2^{-i+1}\right) x_{i}$ and define

$$
\begin{gathered}
C_{i}=\bigcup\left\{B\left(x, 2^{-2 i}\right) \mid x \in\left[x_{i},\left(1+2^{-i}\right) x_{i}\right]\right\}, \\
R_{i}=B\left(y_{i}, 2^{-i}\right) \text { and } D=B(0,1) \cup \bigcup_{i=1}^{\infty}\left(C_{i} \cup R_{i}\right) .
\end{gathered}
$$

Thus $D$ consists of a central room $B(0,1)$ and a sequence of rooms $\left\{R_{i}\right\}$ connected to $B(0,1)$ by narrow corridors $\left\{C_{i}\right\}$.

General rooms and corridors type domains such as $D$ are studied in detail in $\S 10$. We could apply Theorem 15 from that section to easily see that $M_{2}(D)<$ $\infty$ and that the imbedding $W^{1,2}(D) \rightarrow L^{2}(D)$ is not compact. Our goal now, however, is to show that the hypotheses of Theorem 8 do not imply that such an imbedding is compact. We also wish to demonstrate how Theorem 8 can be used to show that a specific domain is a $p$-Poincare domain. Accordingly, we proceed to show that $D$ satisfies the hypotheses of Theorem 8 .

To each point $x \in D$ we associate an arc $\gamma_{x}$ in $D$ with initial point 0 and terminal point $x$. These arcs will be used to determine the sets $P(Q)$ in this example. The arc associated with $x \in B(0,1)$ traces the line segment $[0, x]$. For $x \in C_{i} \backslash\left\{B(0,1) \cup R_{i}\right\}$ let $\tilde{x}$ be the point on $\left[0,\left(1+2^{-i}\right) x_{i}\right]$ which is closest to $x$, and define $\gamma_{x}$ to be the arc that traces the line segments $[0, \tilde{x}]$ followed by $[\tilde{x}, x]$. For $x \in R_{i}$ we define $\gamma_{x}$ so that it traces the line segments $\left[0, y_{i}\right]$ followed by $\left[y_{i}, x\right]$. This completes the description of the family of arcs.

Let $W$ be a Whitney decomposition for $D$. For $Q \in W$, denote by $\gamma_{Q}$ the arc from our family of arcs associated with $x_{Q}$, the center of $Q$. Define $P(Q)$ to be the union of all cubes in $W$ that intersect $\gamma_{Q}$. We shall show that $D$ is a 2-Poincare domain by applying Theorem 8 with $\lambda=1 / 4$ and this definition of the sets $P(Q)$. Thus it suffices to show that

$$
\sup _{Q \in W} \frac{1}{d(Q)} \int_{S(Q)} \int_{P\left(Q_{x}\right)} \frac{d y}{\delta_{D}(y)^{3}} d x<\infty .
$$

We sketch a proof that (9.1) holds. It is easily checked that if $x \in D$, then

$$
\int_{\gamma_{x}} \frac{d s}{\delta_{D}(y)} \preceq k_{D}(0, x) \text {. }
$$

Thus, by Lemma 9 , it suffices to show that

$$
\sup _{Q \in W} \frac{1}{d(Q)} \int_{S(Q)} k_{D}(0, x) d x<\infty .
$$

First consider a cube $Q \subset C_{i} \cup R_{i}$ with the property that the diameter of $S(Q)$ satisfies $d(S(Q)) \leq 10 \delta_{D}\left(x_{Q}\right)$. An easy estimate shows that for such a 
cube $k_{D}\left(0, x_{Q}\right) \preceq 2^{i}+\log (1 / d(Q))$, so $m(Q) \cdot k_{D}\left(0, x_{Q}\right) \preceq d(Q)^{2}$. Also it is easily seen that

$$
\int_{S(Q)} k_{D}\left(x_{Q}, x\right) d x \preceq d(Q)^{2} \int_{0}^{d(Q)} \log \frac{1}{t} d t \preceq d^{2}(Q) .
$$

Thus

$$
\begin{aligned}
\frac{1}{d(Q)} \int_{S(Q)} k_{D}(0, x) d x & \leq \frac{1}{d(Q)}\left(m(S(Q)) \cdot k_{D}\left(0, x_{Q}\right)+\int_{S(Q)} k_{D}\left(x_{Q}, x\right) d x\right) \\
& \preceq 1 .
\end{aligned}
$$

A similar computation establishes that the same estimate holds for a cube $Q \subset C_{i} \cup R_{i}$ with the property that $d(S(Q)) \geq 10 \delta_{D}\left(x_{Q}\right)$. The key point is showing that

$$
\int_{C_{i} \cup R_{i}} k_{D}\left(x_{i}, x\right) d x \preceq \int_{R_{i}} k_{D}\left(x_{i}, x\right) d x \preceq 2^{-2 i} .
$$

Thus we have shown that if $Q \subset C_{i} \cup R_{i}$, then

$$
\frac{1}{d(Q)} \int_{S(Q)} k_{D}(0, x) d x \preceq 1 \text {. }
$$

We now consider a cube $Q \subset B(0,1)$. As above we have that

$$
\frac{1}{d(Q)} \int_{S(Q) \cap B(0,1)} k_{D}(0, x) d x \preceq 1 .
$$

Also, since $\left\{B\left(x_{i}, 2^{-i}\right)\right\}$ are pairwise disjoint,

$$
\sum_{R_{i} \subset S(Q)} 2^{-2 i} \preceq d(Q)^{2} .
$$

This with (9.3) shows

$$
\begin{aligned}
\int_{S(Q) \backslash B(0,1)} k_{D}(0, x) d x & \leq \sum_{R_{i} \subset S(Q)}\left(k_{D}\left(0, x_{i}\right) \cdot m\left(C_{i} \cup R_{i}\right)+\int_{C_{i} \cup R_{i}} k_{D}\left(x_{i}, x\right) d x\right) \\
& \preceq \sum_{R_{i} \subset S(Q)}\left(i 2^{-3 i}+2^{-2 i}\right) \\
& \preceq d(Q)^{2} .
\end{aligned}
$$

This estimate together with (9.5) shows that if $Q \subset B(0,1)$, then

$$
\frac{1}{d(Q)} \int_{S(Q)} k_{D}(0, x) d x \preceq 1 .
$$

Now (9.2) follows from this and (9.4), so we have shown that $M_{2}(D)<\infty$.

To finish this example we now need to demonstrate that the imbedding $W^{1,2}(D) \rightarrow L^{2}(D)$ is not compact. For $1 \leq i<\infty$, define

$$
u_{i}(x)= \begin{cases}0, & x \in D \backslash\left(C_{i} \cup R_{i}\right), \\ 2^{5 i / 2}(|x|-1), & x \in C_{i} \cap B\left(0,1+2^{-i}\right), \\ 2^{3 i / 2}, & x \in\left(R_{i} \cup C_{i}\right) \backslash B\left(0,1+2^{-i}\right) .\end{cases}
$$


A straightforward calculation shows that $\left\|u_{i}\right\|_{L^{2}(D)} \approx 1$ and $\left\|u_{i}\right\|_{W^{1,2}(D)} \approx 1$, for all $i$.

If the imbedding $W^{1,2}(D) \rightarrow L^{2}(D)$ were compact it would follow that there is a subsequence of $\left\{u_{i}\right\}$ converging to $u \in L^{2}(D)$ in norm. But then $\|u\|_{L^{2}(D)} \approx 1$, which is a contradiction since $u_{i}(x) \rightarrow 0$ almost everywhere. Hence the imbedding $W^{1,2}(D) \rightarrow L^{2}(D)$ is not compact, and we are done with the example.

\section{ROOMS AND CORRIDORS TYPE EXAMPLES}

It is a well-known elementary fact that a finite union of Poincaré domains is again a Poincaré domain. In this section we take up the study of infinite unions of Poincaré domains. A "rooms and corridors" type domain consists of a central cube shaped room along with an infinite disjoint collection of cube shaped rooms which are connected to the central room by narrow corridors (or tubes if $d>2$ ), such as the domain constructed in $\S 9$. The resulting domain may or may not have $M_{p}$ finite. The use of rooms and corridors type examples in the study of the Poincare inequality can found in $[\mathrm{CH}],[\mathrm{M}],[\mathrm{S}]$ and $[\mathrm{SS} 2]$, and variants of these domains are used in [Am] and [AS].

In this section, we characterize those rooms and corridors type examples which are $p$-Poincaré domains by using the $k_{p}$ metric introduced in $\S 7$. We then construct a specific example to show that the condition $p \geq d$ in Theorem 1 is necessary. Results on compact imbeddings of rooms and corridors type domains are also obtained. The theorems in this section again demonstrate the important relationship between the $k_{p}$ metric and the $p$-Poincare inequality. Some of these results generalize earlier results of ours in [SS2].

Definition. Let $T$ be a domain in $R^{d}$. For $x_{1}, x_{2} \in T$ and $1<p<\infty$, we define $h_{p, T}\left(x_{1}, x_{2}\right)=k_{p, T}^{p-1}\left(x_{1}, x_{2}\right)$. For $p=1$, we put

$$
h_{1, T}\left(x_{1}, x_{2}\right)=\inf \left\{\sup _{x \in \gamma} \delta_{T}^{1-d}(x) \mid \gamma \text { is a path from } x_{1} \text { to } x_{2} \text { in } T\right\} \text {. }
$$

This definition is motivated by the fact that for a fixed arc $\gamma$ in $T$,

$$
\lim _{p \rightarrow 1}\left(\int_{\gamma} \frac{d s}{\delta_{T}(y)^{\frac{d-1}{p-1}}}\right)^{p-1}=\sup _{y \in \gamma} \delta_{T}^{1-d}(y) .
$$

Lemma 11. Let $T$ be a domain in $R^{d}$. Suppose that $W$ is a Whitney decomposition of $T$ and that $1 \leq p<\infty$. Then

$$
\left|u_{Q_{1}}-u_{Q_{2}}\right|^{p} \preceq h_{p, T}\left(x_{1}, x_{2}\right) \cdot \int_{T}|\nabla u|^{p} d x
$$

whenever $Q_{1}, Q_{2} \in W$, with $x_{i} \in Q_{i}$ for $i=1,2$, and $u \in C^{1}(T)$. 
Proof. For $p>1$, let $\gamma$ be an arc in $T$ joining $x_{1}$ to $x_{2}$ and satisfying

$$
\int_{\gamma} \frac{d s}{\delta_{T}(y)^{\frac{d-1}{p-1}}} \leq 2 k_{p, T}\left(x_{1}, x_{2}\right) .
$$

Let $P$ be the collection of Whitney cubes in $W$ which intersect $\gamma$. By Lemmas 8 and 9 and (10.3) we obtain that

$$
\begin{aligned}
\left|u_{Q_{1}}-u_{Q_{2}}\right|^{p} & \leq\left(\int_{P} \frac{|\nabla u|}{\delta_{T}(y)^{d-1}}\right)^{p} \\
& \leq \int_{P}|\nabla u|^{p} d x \cdot\left(\int_{P} \frac{d y}{\delta_{T}(y)^{p^{\prime}(d-1)}}\right)^{p-1} \\
& \preceq k_{p, T}^{p-1}\left(x_{1}, x_{2}\right) \cdot \int_{P}|\nabla u|^{p} d x \\
& =h_{p, T}\left(x_{1}, x_{2}\right) \cdot \int_{P}|\nabla u|^{p} d x
\end{aligned}
$$

which verifies (10.2) in this case. An analogous argument for the case $p=1$ establishes (10.2) for all $1 \leq p<\infty$. This completes the proof.

See [SS2] where this lemma is proved for the special case of simply connected planar domains with $p=2$ and $u$ an analytic function on $T$.

We now consider a more general configuration of a connected domain $D \subset$ $R^{d}$ with finite volume. We assume that $D=\bigcup_{n=0}^{\infty} G_{n}$ where each $G_{n}$ is an open connected subdomain of $D$. Moreover, we assume that $\left\{G_{n}\right\}_{n=1}^{\infty}$ is a disjoint collection and that $G_{n} \cap G_{0}$ is a nonempty set for each $n$. Corresponding to each region $G_{n}$, with $n \geq 1$, there is a subregion $\tilde{G}_{n}$ of $G_{0}$ for which $G_{n} \cap \tilde{G}_{n}$ is a nonempty set. Put $T_{n}=G_{n} \cup \tilde{G}_{n}$ for $n \geq 1$, so that $T_{n}$ is an open connected subdomain of $D$. Using the construction on page 167 of Stein's book [St], we construct a Whitney decompositions $W_{n}$ of $T_{n}$ with defining parameters independent of $n$. Finally, assume that $Q_{n}, \tilde{Q}_{n} \in W_{n}$ with $Q_{n} \subset G_{n}, \tilde{Q}_{n} \subset \tilde{G}_{n}$ and that $x_{n}, \tilde{x}_{n}$ are the centers of $Q_{n}, \tilde{Q}_{n}$.

Theorem 13. Let $1 \leq p<\infty$ and let $M, K$ be finite constants. If

(a) $m\left(G_{n}\right), m\left(Q_{n}\right), m\left(\tilde{G}_{n}\right)$ and $m\left(\tilde{Q}_{n}\right)$ are all comparable for each $n \geq 1$, with constants independent of $n$,

(b) $\left\{\tilde{Q}_{n}\right\}_{n \geq 1}$ is disjoint and

$$
\sup _{x \in D} \sum_{n=1}^{\infty} \chi_{T_{n}}(x) \leq K
$$

(c) $M_{p}^{p}\left(G_{n}\right) \leq M$ for all $n \geq 0$ and

(d) $h_{p, T_{n}}\left(\tilde{x}_{n}, x_{n}\right) \cdot m\left(G_{n}\right) \leq M$ for all $n \geq 1$,

then $M_{p}(D)<\infty$. 
Proof. Let $u$ be a function defined on $D$ satisfying $\nabla u \in L^{p}(D)$ and $u \equiv 0$ on $Q_{0}$. For $n \geq 1$, we use (a) to compute that

$$
\begin{aligned}
\int_{G_{n}}|u|^{p} d x \leq & \int_{G_{n}}\left|u-u_{G_{n}}\right|^{p} d x+\left|u_{G_{n}}-u_{Q_{n}}\right|^{p} m\left(Q_{n}\right) \\
& +\left|u_{Q_{n}}-u_{\tilde{Q}_{n}}\right|^{p} m\left(G_{n}\right)+\left|u_{\tilde{Q}_{n}}\right|^{p} m\left(\tilde{Q}_{n}\right) \\
\leq & 2 \int_{G_{n}}\left|u-u_{G_{n}}\right|^{p} d x+\int_{\tilde{Q}_{n}}|u|^{p} d x+\left|u_{Q_{n}}-u_{\tilde{Q}_{n}}\right|^{p} m\left(G_{n}\right) \\
& =2 \cdot A_{n}+B_{n}+C_{n} .
\end{aligned}
$$

By (c), we have that

$$
\sum_{n=1}^{\infty} A_{n} \leq M \sum_{n=1}^{\infty} \int_{G_{n}}|\nabla u|^{p} d x \leq M \int_{D}|\nabla u|^{p} d x .
$$

Since $u \equiv 0$ on $Q_{0}$ it follows from Lemma 5 and conditions (b), (c) that

$$
\sum_{n=1}^{\infty} B_{n} \leq \int_{G_{0}}|u|^{p} d x \preceq M \int_{D}|\nabla u|^{p} d x .
$$

Combining these estimates we obtain that

$$
\int_{D}|u|^{p} d x \leq \sum_{n=0}^{\infty} \int_{G_{n}}|u|^{p} d x \preceq M \int_{D}|\nabla u|^{p} d x+\sum_{n=1}^{\infty} C_{n} .
$$

Using Lemma 11 we obtain that

$$
\left|u_{Q_{n}}-u_{\tilde{Q}_{n}}\right|^{p} \preceq h_{p, T_{n}}\left(\tilde{x}_{n}, x_{n}\right) \cdot \int_{T_{n}}|\nabla u|^{p} d x, \quad n \geq 1 .
$$

Finally, we have by conditions (b), (d) and (10.4) that

$$
\begin{aligned}
\sum_{n=1}^{\infty} C_{n} & \leq \sum_{n=1}^{\infty} h_{p, T_{n}}\left(\tilde{x}_{n}, x_{n}\right) \cdot m\left(G_{n}\right) \cdot \int_{T_{n}}|\nabla u|^{p} d x \\
& \leq M \int_{D}|\nabla u|^{p} \sum_{n=1}^{\infty} \chi_{T_{n}} d x \leq M K \int_{D}|\nabla u|^{p} d x,
\end{aligned}
$$

and hence that

$$
\int_{D}|u|^{p} d x \preceq M(K+1) \int_{D}|\nabla u|^{p} d x
$$

whenever $u$ vanishes on $Q_{0}$. Thus, the proof is complete by Lemma 5 .

We now consider an infinite union of domains $\left\{G_{n}\right\}$ with the property that each of the imbeddings $W^{1, p}\left(G_{n}\right) \rightarrow L^{p}\left(G_{n}\right)$ is compact. The assumptions and the notation introduced prior to Theorem 13 are still in force.

Theorem 14. Let $1 \leq p<\infty$ and let $M, K$ be finite constants. If

(a) $m\left(G_{n}\right), m\left(Q_{n}\right), m\left(\tilde{G}_{n}\right)$ and $m\left(\tilde{Q}_{n}\right)$ are all comparable for each $n \geq 1$, with constants independent of $n$, 
(b) $\left\{\tilde{Q}_{n}\right\}_{n \geq 1}$ is disjoint and

$$
\sup _{x \in D} \sum_{n=1}^{\infty} \chi_{T_{n}}(x) \leq K
$$

(c) $\lim _{n \rightarrow \infty} M_{p}^{p}\left(G_{n}\right)=0$,

(d) $\lim _{n \rightarrow \infty} h_{p, T_{n}}\left(\tilde{x}_{n}, x_{n}\right) \cdot m\left(G_{n}\right)=0$,

(e) $\lim _{n \rightarrow \infty} \delta_{D}\left(\tilde{x}_{n}\right)=0$, and

(f) the imbedding $W^{1, p}\left(G_{n}\right) \rightarrow L^{p}\left(G_{n}\right)$ is compact for all $n \geq 0$,

then the imbedding $W^{1, p}(D) \rightarrow L^{p}(D)$ is compact.

Proof. Theorem 11 will be used to show the compactness of the imbedding. As a first step, we show that

$$
\lim _{N \rightarrow \infty} \sup \left\{\sum_{n=N+1}^{\infty} \int_{G_{n}}|u|^{p} d x \mid\|u\|_{W^{1, p}(D)}=1\right\}=\lim _{N \rightarrow \infty} S_{N}=0 .
$$

Arguing as in the proof of Theorem 13, by using (a) we see that

$$
S_{N} \preceq A_{N}^{\prime}+B_{N}^{\prime}+C_{N}^{\prime},
$$

where

$$
\begin{aligned}
& A_{N}^{\prime}=\sup \left\{\sum_{n=N+1}^{\infty} \int_{G_{n}}\left|u-u_{G_{n}}\right|^{p} d x \mid\|u\|_{W^{1, p}(D)}=1\right\}, \\
& B_{N}^{\prime}=\sup \left\{\sum_{n=N+1}^{\infty} \int_{\tilde{Q}_{n}}|u|^{p} d x \mid\|u\|_{W^{1, p}(D)}=1\right\}, \\
& C_{N}^{\prime}=\sup \left\{\sum_{n=N+1}^{\infty}\left|u_{Q_{n}}-u_{\tilde{Q}_{n}}\right|^{p} m\left(G_{n}\right) \mid\|u\|_{W^{1, p}(D)}=1\right\} .
\end{aligned}
$$

By (c) we have that $\lim _{N \rightarrow \infty} A_{N}^{\prime}=0$, since

$$
\int_{G_{n}}\left|u-u_{G_{n}}\right|^{p} d x \leq M_{p}^{p}\left(G_{n}\right) \int_{G_{n}}|u|^{p} d x .
$$

For any $k \geq 1$, by (e) and the fact that $\tilde{Q}_{n} \in W_{n}$, we can pick $N$ such that

$$
\bigcup_{n=N+1}^{\infty} \widetilde{Q}_{n} \subset D \backslash D_{k},
$$

where $D_{k}$ is as in (8.1). Thus by applying (f) with $n=0$ and Theorem 11, we have that $\lim _{N \rightarrow \infty} B_{N}^{\prime}=0$. Now, using (10.4) and (b) as is the proof of Theorem 13, we estimate

$$
\begin{aligned}
C_{N}^{\prime} & \leq \sup \left\{\sum_{n=N+1}^{\infty} h_{p, T_{n}}\left(\tilde{x}_{n}, x_{n}\right) \cdot m\left(G_{n}\right) \cdot \int_{T_{n}}|\nabla u|^{p} d x \mid\|u\|_{W^{1, p}(D)}=1\right\} \\
& \leq K \sup _{n \geq N+1} h_{p, T_{n}}\left(\tilde{x}_{n}, x_{n}\right) \cdot m\left(G_{n}\right) .
\end{aligned}
$$


Thus $\lim _{N \rightarrow \infty} C_{N}^{\prime}=0$, by (d). The proof of (10.6) is now complete.

It is an easy consequence of the definition of a compact imbedding that, since each imbedding $W^{1, p}\left(G_{n}\right) \rightarrow L^{p}\left(G_{n}\right)$ is compact, the imbedding

$$
W^{1, p}\left(\bigcup_{n=0}^{N} G_{n}\right) \rightarrow L^{p}\left(\bigcup_{n=0}^{N} G_{n}\right)
$$

is compact for each $N \geq 0$. (Alternately, one can use Theorem 11 to see this.) Thus by Theorem 11 again, and with $D_{k}$ as in (8.1),

$$
\lim _{k \rightarrow \infty} \sup \left\{\int_{\bigcup_{n=0}^{N} G_{n} \backslash D_{k}}|u|^{p} d x \mid\|u\|_{W^{1, p}\left(G_{N}\right)}=1\right\}=0, \quad N \geq 0 .
$$

But since

$$
D \backslash D_{k} \subset\left(\bigcup_{n=0}^{N} G_{n} \backslash D_{k}\right) \cup\left(\bigcup_{n=N+1}^{\infty} G_{n}\right),
$$

(10.6) and (10.7) imply that

$$
\lim _{k \rightarrow \infty} \sup \left\{\int_{D \backslash D_{k}}|u|^{p} d x \mid\|u\|_{W^{1, p}(D)}=1\right\}=0,
$$

so an application of Theorem 11 completes the proof.

We now proceed to simplify the above geometric configurations. Let $R_{n}$ denote the ball $B\left(x_{n}, c_{n}\right)$ with center $x_{n}$ and radius $c_{n}$, where $n=0,1, \ldots$. We assume that $x_{0}=0, c_{0}=1,1<\left|x_{n}\right|<2, a_{n} \leq c_{n}$ and that the collection of balls $\left\{R_{n}\right\}_{n=0}^{\infty}$ is disjoint. For $n \geq 1$, let $x_{n}^{\prime}=x_{n} /\left|x_{n}\right|, b_{n}=\left|x_{n}-x_{n}^{\prime}\right|-c_{n}$ and $C_{n}=\bigcup\left\{B\left(x, a_{n}\right)|0 \leq| x-x_{n}^{\prime} \mid \leq b_{n}\right\}$. We further assume, for $n \geq 1$, that the sets $\left\{C_{n} \cup R_{n}\right\}$ are disjoint and that $D$ is constructed using the rooms $R_{n}$ and the corridors $C_{n}$, i.e.

$$
D=R_{0} \cup\left(\bigcup_{n=1}^{\infty}\left(R_{n} \cup C_{n}\right)\right) .
$$

Theorem 15. Let $1 \leq p<\infty$ and let $D$ be the domain constructed above.

(i) $M_{p}(D)$ is finite if and only if

$$
\sup _{n} h_{p}\left(x_{0}, x_{n}\right) \cdot m\left(R_{n}\right)<\infty .
$$

(ii) Further suppose that

$$
\sup _{n} \frac{a_{n}^{d-1} b_{n}}{c_{n}^{d}} \preceq 1
$$

Then the imbedding $W^{1, p}(D) \rightarrow L^{p}(D)$ is compact if and only if

$$
\lim _{n \rightarrow \infty} h_{p}\left(x_{0}, x_{n}\right) \cdot m\left(R_{n}\right)=0 .
$$


Remark. The geometric interpretation of (10.9) is that $m\left(C_{n}\right) \preceq m\left(R_{n}\right)$. Without a restriction of this type it is easy to construct a counterexample with a starshaped domain.

Proof. (i) Assume that (10.8) holds. We establish that $M_{p}(D)$ is finite by showing that conditions (a)-(d) in Theorem 13 hold. Let $G_{0}=R_{0} \cup\left(\cup_{n=1}^{\infty} C_{n}\right)$. Then $G_{0}$ is starshaped with respect to the origin and hence is a $p$-Poincaré domain by Theorem 6 . For $n \geq 1$ put $G_{n}=R_{n}$. Then by Theorem 6 again we see that condition (c) holds for some finite constant $M$.

For $n \geq 1$, let $\tilde{R}_{n}, \tilde{C}_{n}$ denote the reflections of the sets $R_{n}, C_{n}$ about the sphere $|x|=1$ in $R^{d}$. We put $\tilde{G}_{n}=\tilde{R}_{n} \cup \tilde{C}_{n} \cup C_{n}$ and construct a Whitney decomposition $W_{n}$ of $T_{n}=G_{n} \cup \tilde{G}_{n}$ with constant defining parameters. Finally, we put $Q_{n}$ to be a cube in $W_{n}$ containing $x_{n}$ and let $\tilde{Q}_{n}$ be a cube in $W_{n}$ containing the reflection $\tilde{x}_{n}$ of $x_{n}$. Clearly, conditions (a) and (b) hold, with $K=1$.

To prove that condition (d) holds, we will use the symmetry of $T_{n}$. For $p>1$, let $\Gamma_{n}$ be an arc in $D$ joining $x_{0}$ to $x_{n}$ so that

$$
\int_{\Gamma_{n}} \frac{d s}{\delta_{D}(y)^{\frac{d-1}{p-1}}} \leq 2 k_{p}\left(x_{0}, x_{n}\right) .
$$

Let $\gamma_{n}$ be the subarc of $\Gamma_{n}$ starting from the last exit from $R_{0}$ to the endpoint $x_{n}$. Let $\tilde{\gamma}_{n}$ be the reflection of $\gamma_{n}$ about the sphere $|x|=1$. Clearly, $\tilde{\gamma}_{n} \cup \gamma_{n}$ is an arc in $T_{n}$ joining $\tilde{x}_{n}$ to $x_{n}$. Hence by symmetry,

$$
\begin{aligned}
k_{p, T_{n}}\left(x_{n}, \tilde{x}_{n}\right) & \leq \int_{\tilde{\gamma}_{n} \cup \gamma_{n}} \frac{d s}{\delta_{T_{n}}(y)^{\frac{d-1}{p-1}}} \preceq \int_{\gamma_{n}} \frac{d s}{\delta_{T_{n}}(y)^{\frac{d-1}{p-1}}} \\
& =\int_{\gamma_{n}} \frac{d s}{\delta_{D}(y)^{\frac{d-1}{p-1}}} \leq \int_{\Gamma_{n}} \frac{d s}{\delta_{D}(y)^{\frac{d-1}{p-1}}} \leq 2 k_{p}\left(x_{0}, x_{n}\right) .
\end{aligned}
$$

Thus, condition (d) holds for the case $1<p<\infty$. A similar argument yields (d) in case $p=1$. Hence $M_{p}(D)$ is finite by Theorem 13 .

To prove the converse suppose that $M_{p}(D)$ is finite. We must find a bound $M<\infty$ so that

$$
h_{p}\left(x_{0}, x_{n}\right) \cdot m\left(R_{n}\right) \leq M
$$

holds for all $n$. Fix $n$ and let $\gamma_{n}$ be the line segment $\left[x_{0}, x_{n}\right]$. Integration over $\gamma_{n}$ gives an upper bound for $k_{p}\left(x_{0}, x_{n}\right)$.

We first assume that $1<p<d$. If $b_{n}+c_{n}<2 a_{n}$, then

$$
k_{p}^{p-1}\left(x_{0}, x_{n}\right) \cdot m\left(R_{n}\right) \preceq\left(a_{n}^{1-\frac{d-1}{p-1}}\right)^{p-1} \cdot a_{n}^{d}=a_{n}^{p} \leq 1
$$

and so (10.13) holds with some $M$ which is independent of $n$. If $b_{n}+c_{n} \geq 2 a_{n}$, we construct a function $u_{n}$ which vanishes on $D$ except for points in $C_{n} \cup R_{n}$. 
For $x \in C_{n} \cup R_{n}$, we define

$$
u_{n}(x)=\min \left(1, \frac{\left|x-x_{n}^{\prime}\right|-a_{n}}{2 a_{n}+b_{n}}\right), \quad\left|x-x_{n}^{\prime}\right| \geq a_{n} .
$$

Clearly, $u_{n}=1$ on at least half of $R_{n}$ and hence

$$
m\left(R_{n}\right) \preceq \int_{R_{n}}\left|u_{n}\right|^{p} d x \leq M_{p}^{p}(D) \int_{D}\left|\nabla u_{n}\right|^{p} d x .
$$

If $b_{n} \leq a_{n}$, then it is easily seen that $k_{p}^{p-1}\left(x_{0}, x_{n}\right) \preceq a_{n}^{p-d}$ and $\left|\nabla u_{n}\right| \preceq a_{n}^{-1}$. Thus,

$$
\int_{D}\left|\nabla u_{n}\right|^{p} d x \preceq \frac{m\left(\left\{\left|\nabla u_{n}\right| \neq 0\right\}\right)}{a_{n}^{p}} \preceq a_{n}^{d-p} \preceq k_{p}^{1-p}\left(x_{0}, x_{n}\right) .
$$

Thus, (10.13) holds in this case.

If $b_{n} \geq a_{n}$, then $k_{p}^{p-1}\left(x_{0}, x_{n}\right) \preceq b_{n}^{p-1} / a_{n}^{d-1}$ and $\left|\nabla u_{n}\right| \preceq b_{n}^{-1}$. Again,

$$
\int_{D}\left|\nabla u_{n}\right|^{p} d x \preceq \frac{m\left(\left\{\left|\nabla u_{n}\right| \neq 0\right\}\right)}{b_{n}^{p}} \preceq \frac{b_{n} a_{n}^{d-1}}{b_{n}^{p}} \preceq k_{p}^{1-p}\left(x_{0}, x_{n}\right)
$$

and so (10.13) holds. We have therefore completed the proof for the case $1<p<d$.

For $d<p$, the same proof works, only the estimates are more elementary since the integral

$$
\int_{0}^{1} \frac{d t}{t^{\frac{d-1}{p-1}}}<\infty
$$

is convergent. The case $p=1$ can also be done with a similar argument. Finally, the case $p=d$ requires a slightly different construction for $u_{n}$. If $b_{n} \geq a_{n}$, then the proof is as before. But if $b_{n} \leq a_{n}$, then we define

$$
u_{n}(x)=\min \left(1, \frac{\log \left(\left|x-x_{n}^{\prime}\right| / a_{n}\right)}{\log \left(\left(2 a_{n}+b_{n}\right) / a_{n}\right)}\right), \quad\left|x-x_{n}^{\prime}\right| \geq a_{n},
$$

whenever $x \in C_{n} \cup R_{n}$. Analogous estimates show that (10.13) holds in this case also and the proof of part (i) is complete.

(ii) Assume that (10.10) holds. We will use Theorem 14 to show that the imbedding $W^{1, p}(D) \rightarrow L^{p}(D)$ is compact. Define $G_{0}=R_{0}$, and for $n \geq 1$, $G_{n}=R_{n} \cup C_{n}$ and $\tilde{G}_{n}=\tilde{R}_{n} \cup \tilde{C}_{n}$, where $\tilde{R}_{n}$ and $\tilde{C}_{n}$ are as in the proof of (i). Let $Q_{n}, \tilde{Q}_{n}, x_{n}$ and $\tilde{x}_{n}$ also be defined as in the proof of (i). Using (10.9) and estimating $k_{p}\left(x_{0}, x_{n}\right)$ as in (i), we get that

$$
b_{n}^{p} \preceq b_{n}^{p-1} c_{n}^{d} / a_{n}^{d-1} \preceq h_{p}\left(x_{n}, \tilde{x}_{n}\right) \cdot m\left(R_{n}\right) .
$$

Hence $b_{n} \rightarrow 0$, by $(10.10)$ and (10.12). We also have that $c_{n} \rightarrow 0$ since $m(D)<\infty$.

As in the proof of (i), conditions (a) and (b) of Theorem 14 are immediate by the definition of $Q_{n}, \tilde{Q}_{n}$ and $x_{n}$. Condition (c) holds as a consequence 
of Theorem 7, since $b_{n}+c_{n} \rightarrow 0$. Condition (d) is immediate from (10.10) and (10.12), while condition (e) follows since $b_{n}+c_{n} \rightarrow 0$. Finally, it is well known that the imbeddings in condition (f) are compact. See Chapter 6 of [Ad], for example, or alternately Corollary 6 could be used. Thus the imbedding $W^{1, p}(D) \rightarrow L^{p}(D)$ is compact by Theorem 14 .

To prove the converse, suppose that (10.10) fails. By passing to a subsequence, assume without loss of generality that $\inf _{n} h_{p}\left(x_{0}, x_{n}\right) \cdot m\left(R_{n}\right) \succeq 1$. Thus

$$
\left(\left(\log \frac{1}{a_{n}}\right)^{p-1}+\frac{b_{n}^{p-1}}{a_{n}^{d-1}}\right) \cdot c_{n}^{d} \succeq 1, \quad n \geq 1 .
$$

First consider the case that $\lim \sup b_{n}^{p-1} c_{n}^{d} / a_{n}^{d-1} \succeq 1$. By passing to a subsequence again, without lossed generality assume that

$$
b_{n}^{p-1} c_{n}^{d} / a_{n}^{d-1} \succeq 1, \quad n \geq 1 .
$$

To finish the proof of this case, we demonstrate that the imbedding $W^{1, p}(D)$ $\rightarrow L^{p}(D)$ is not compact in a manner similiar to that used in $\S 9$. For $1 \leq n \leq$ $\infty$, define

$$
u_{n}(x)= \begin{cases}0, & x \in D \backslash G_{n}, \\ b_{n}^{-1} c_{n}^{-d / p}(|x|-1), & x \in C_{n} \backslash\left\{1<|x|<1+b_{n}\right\}, \\ c_{n}^{-d / p}, & x \in R_{n} \backslash B\left(0,1+b_{n}\right) .\end{cases}
$$

A straightforward calculation shows that $\left\|u_{n}\right\|_{L^{2}(D)} \approx 1$ and $\left\|\nabla u_{n}\right\|_{L^{p}(D)} \preceq 1$, for all $n$. (10.14) was used in the second estimate.

If the imbedding $W^{1, p}(D) \rightarrow L^{p}(D)$ were compact, it would follow that there is a subsequence of $\left\{u_{n}\right\}$ converging to $u \in L^{p}(D)$ in norm. This is a contradiction, since it would follow that $\|u\|_{L^{p}(D)} \approx 1$, but $u_{n}(x) \rightarrow 0$ for all $x \in D$.

The final case, with $\lim \sup \left(\log \frac{1}{a_{n}}\right)^{p-1} \cdot c_{n}^{d} \succeq 1$, is handled similarly, with the functions defined as in the proof of (ii). This completes the proof of Theorem 15.

Example. Fix $m \geq 1$ and let $D_{m}$ be constructed by attaching to the unit ball in $R^{d}$ a disjoint sequence of balls, of radius $c_{n}=2^{-n}$, and connecting tubes of length $b_{n}=\left(c_{n}\right)^{-m}$ and radius $a_{n}=b_{n} / n$. It is easily seen that condition (1.2) holds since

$$
\sup _{n}\left(\frac{b_{n}}{a_{n}}-\log b_{n}\right) \cdot \frac{1}{\log c_{n}^{-1}}=\frac{1+m \log 2}{\log 2}<\infty
$$

and hence $D_{m}$ is a Hölder domain. Thus, Theorem 1 shows that $M_{p}\left(D_{m}\right)$ is finite for all $d \leq p<\infty$. 
On the other hand, Theorem 15(i) implies that for $1 \leq p<d, M_{p}\left(D_{m}\right)$ is finite if and only if

$$
\sup _{n} \frac{b_{n}^{p-1}}{a_{n}^{d-1}} \cdot c_{n}^{d}<\infty .
$$

Since the supremum in (10.15) is clearly infinity for $p \leq d(1-1 / m)$, we see that $M_{p}\left(D_{m}\right)=\infty$ for this range of $p$ 's. Thus, the range of $p$ in Theorem 1 is best possible.

\section{REFERENCES}

[Ad] R. A. Adams, Sobolev spaces, Academic Press, New York, 1975.

[Am] C. J. Amick, Some remarks on Rellich's theorem and the Poincaré inequality J. London Math. Soc. 18 (1978), 81-93.

[AS] S. Axler and A. Shields, Univalent multipliers of the Dirichlet space, Michigan Math. J. 32 (1985), 65-80.

[BP] J. Becker and C. Pommerenke, Hölder continuity of conformal mappings and non-quasiconformal Jordan curves, Comment. Math. Helv. 57 (1982), 221-225.

[GM] F. W. Gehring and O. Martio, Lipschitz classes and quasiconformal mappings, Ann. Acad. Sci. Fenn. Ser. A I Math. 10 (1985), 203-219.

[GO] F. W. Gehring and B. G. Osgood, Uniform domains and the quasi-hyperbolic metric, J. Analyse Math. 36 (1979), 50-74.

[GP] F. W. Gehring and B. P. Palka, Quasiconformally homogeneous domains, J. Analyse Math. 30 (1976), 172-199.

[GT] D. Gilbarg and N. S. Trudinger, Elliptic partial differential equations of second order, Springer-Verlag, Berlin and Heidelberg, 1983.

[H] D. H. Hamilton, On the Poincaré inequality, Complex Variables Theory Appl. 5 (1986), 265-270.

[J1] P. Jones, Extensions theorems for BMO, Indiana Univ. Math. J. 29 (1980), 41-66.

[J2] _ Quasiconformal mappings and extensions of functions in Sobolev space, Acta Math. 1-2 (1981), 71-88.

[Ma] G. J. Martin, Quasiconformal and bi-Lipschitz homeomorphisms, uniform domains and the quasihyperbolic metric, Trans. Amer. Math. Soc. 292 (1985), 169-191.

[Mar] O. Martio, John domains, bilipschitz balls and Poincaré inequality, Rev. Roumaine Math. Pures Appl. 33 (1988), 107-112.

[MV] O. Martio and M. Vourinen, Whitney cubes, p-capacity, and Minkowski content, Expo. Math. 5 (1987), 17-40.

[M] V. G. Maz' ja, Sobolev spaces, Springer-Verlag, Berlin and Heidelberg, 1985.

[MS] N. Meyers and J. Serrin, $H=W$, Proc. Nat. Acad. Sci. U.S.A. 51 (1964), 1055-1056.

[Mu] B. Muckenhoupt, Weighted norm inequalities for the Hardy maximal function, Trans. Amer. Math. Soc. 165 (1972), 207-226.

[R] F. Rellich, Ein Satz über mittlere Konvergenz, Göttingen Nachr. (1930), 30-35.

[SS1] W. Smith and D. A. Stegenga, A geometric characterization of Hölder domains, J. London Math. Soc. 35 (1987), 471-480.

[SS2] _ Poincare domains in the plane, Proceedings of R. Nevanlinna Colloq., 1987.

[S] S. G. Staples, $L^{p}$ averaging domains and the Poincaré inequality, Ann. Acad. Sci. Fenn. (to appear).

[St] E. M. Stein, Singular integrals and differentiability properties of functions, Princeton University Press, Princeton, N.J., 1970.

Department of Mathematics, University of Hawail, Honolulu, Hawail 96822 